\title{
Complete Analyticity for 2D Ising Completed
}

\author{
Roberto H. Schonmann ${ }^{1}$, Senya B. Shlosman ${ }^{2}$ \\ ${ }_{1}^{1}$ Mathematics Department University of California at Los Angeles Los Angeles, CA 90024, USA \\ 2 Mathematics Department University of California at Irvine Irvine, CA 92717, USA and Institute \\ for the Information Transmission Problems Russian Academy of Sciences Moskow, Russia
}

Received: 1 August 1994

\begin{abstract}
We study the behavior of the two-dimensional nearest neighbor ferromagnetic Ising model under an external magnetic field $h$. We extend to every subcritical value of the temperature a result previously proven by Martirosyan at low enough temperature, and which roughly states that for finite systems with boundary conditions under a positive external field, the boundary effect dominates in the bulk if the linear size of the system is of order $B / h$ with $B$ small enough, while if $B$ is large enough, then the external field dominates in the bulk. As a consequence we are able to complete the proof that "complete analyticity for nice sets" holds for every value of the temperature and external field in the interior of the uniqueness region in the phase diagram of the model.

The main tools used are the results and techniques developed to study large deviations for the block magnetization in the absence of the magnetic field, and recently extended to all temperatures below the critical one by Ioffe.
\end{abstract}

\section{Introduction}

In this paper we consider the two-dimensional ferromagnetic Ising model with homogeneous interaction between nearest neighbors and subject to a homogeneous external field, i.e., the statistical mechanics model on $\mathbb{Z}^{2}$ with formal Hamiltonian

$$
H_{h}(\sigma)=-\frac{1}{2} \sum_{x, y \text { n.n. }} \sigma(x) \sigma(y)-\frac{h}{2} \sum_{x} \sigma(x),
$$

where $\sigma(x)= \pm 1$ is the spin at the site $x \in \mathbb{Z}^{2}$, and the first sum runs over pairs of sites which are nearest neighbors in $\mathbb{Z}^{2}$, each pair counted only once. Precise definitions, along with notation will be provided in the next section, but below we describe in somewhat informal terms, what will be done.

The work of R.H.S was partially supported by the N.S.F. through grants DMS 9100725 and DMS 9400644 and that of S.B.S. by the N.S.F. through grant DMS 9208029 and by the Russian Fund for Fundamental Research through grant 930101470 
We will be considering Gibbs measures for this model on various subsets of the lattice (including the full lattice) at temperature $T$, and we will be mostly concerned with the case in which the temperature is below the critical value $T_{c}$ and the external field $h$ is different from 0 , but small. In this case, the system is known to be in a "single phase regime": there is a unique Gibbs measure in the infinite volume, and in particular any boundary effect in the bulk of a finite subset of the lattice vanishes as this subset grows and covers the lattice. The behavior is nevertheless expected to be delicate, since we may be close simultaneously to the phase coexistence line and to the critical point. Indeed, some results which were proven before in case the temperature is low enough, or the external field is strong enough, or the temperature is above the critical value (and the external field takes any value) seem harder to prove in case we are close to the critical point and $T<T_{c}$. Specifically, we have in mind two results. One of them due originally to Martirosyan in [Mar], and strengthened in [Sch2], states that for low $T$, the critical linear size of a box such that the boundary condition is still felt in the bulk is of order $1 /|h|$ as $h$ vanishes. The other one is what we propose to call "complete analyticity for nice sets" and which in [MO1] and [MO2] was implicitly called "strong mixing for cubes," and proved in $[\mathrm{MOS}]$ to hold in most of the uniqueness region in the phase diagram $h \times T$, except precisely for the region that concerns us here, but where it was also believed to hold. This very strong type of mixing property is by now known to be equivalent to several desirable properties of the Gibbs measure in the form of estimates which are uniform on a large class of finite subsets of the lattice, including all squares. One of these properties is a uniform upper bound on the logarithmic Sobolev constant related to the Dirichlet form associated to the Gibbs measures. Morally speaking one can say that when "complete analyticity for nice sets" holds the system is as well behaved as if it were in a regime of weak coupling, i.e., high temperature or strong magnetic field.

In this paper we will provide proofs that extend the results of [Mar, Sch2 and MOS], alluded to above, to the full part of the phase diagram where they were expected to hold. Surprisingly maybe, the main new tool that allowed us to do so concerns the Ising model in the absence of the external field. This tool is the result (and also techniques) by Ioffe [Iof 1, Iof 2] on second order larger deviations in the regime $T<T_{c}$, in which the rate function is computed exactly in terms of the Wulff shape associated to the surface tension. Such a result had been derived in $[\mathrm{DKS}]$ and rederived in [Pfi] for low enough $T$, using in part cluster expansions. One way to understand the fact that large deviation results for the regime in which $h=0$ are being used to analyze the system under a small magnetic field is to think of the latter as a perturbation of the former. The perturbation is locally (at each site of the lattice) small, but acts globally and for this reason changes completely the character of the set of Gibbs measures (only one phase is left); there is then a size, which turns out to be of order $1 / h$, for which the perturbation due to the external field competes on equal footing with the boundary effect. The exact large deviation estimates for the unperturbed system allow one to estimate the effect of the perturbation. In the language sometimes used by people who work on large deviations, the Gibbs measure under the external field is obtained from the one without the external field by "tilting" it. One advantage of being able to carry out this project is that the new results that we prove are not in the form of new large deviation estimates, but rather are theorems which describe the typical behavior of the system in certain regimes; in other words, we are not looking at the most likely among unlikely scenarios, but at typical scenarios. This feature of the work stresses 
once again that one of the motivations for studying large deviations is in order to analyze the probable behavior under "tilted distributions."

In the next section we will introduce most of the necessary notation and terminology. The results will be presented in Sect. 3, after we review the large deviation estimates which served as a basis for the new derivations; we will label the statements of the results that will be used as tools with letters and the statements of the new results with numbers. The proofs of the technical results will be presented in Sect. 4. In the appendix, a simplified proof of an estimate due to Ioffe will be presented.

\section{Notation and Terminology}

The lattice. The cardinality of a set $\Gamma \subset \mathbb{Z}^{2}$ will be denoted by $|\Gamma|$. The expression $\Gamma \subset \subset \mathbb{Z}^{2}$ will mean that $\Gamma$ is a finite subset of $\mathbb{Z}^{2}$. For each $x \in \mathbb{Z}^{2}$, we define the usual norms $\|x\|_{p}=\left(\left|x_{1}\right|^{p}+\left|x_{2}\right|^{p}\right)^{1 / p}, \quad p>0$ finite, and $\|x\|_{\infty}=\max \left\{\left|x_{1}\right|,\left|x_{2}\right|\right\}$. The distance between two sets $A, B \in \mathbb{Z}^{2}$ in each one of these norms will be denoted by

$$
\operatorname{dist}_{p}(A, B)=\inf \left\{\|x-y\|_{p}: x \in A, y \in B\right\} .
$$

In case $A=\{x\}$, we also write $\operatorname{dist}_{p}(A, B)=\operatorname{dist}_{p}(x, B)$. The interior and exterior boundaries of a set $\Gamma \subset \mathbb{Z}^{2}$ will be denoted, respectively by

$$
\partial_{\text {int }} \Gamma=\left\{x \in \Gamma:\|x-y\|_{1}=1 \text { for some } y \notin \Gamma\right\},
$$

and

$$
\partial_{\mathrm{ext}} \Gamma=\left\{x \notin \Gamma:\|x-y\|_{1}=1 \text { for some } y \in \Gamma\right\} .
$$

For lattice squares centered at the origin, we will use the notation

$$
\Lambda(l)=\mathbb{Z}^{2} \cap[-l / 2, l / 2]^{2} .
$$

The set of bonds, i.e., (unordered) pairs of nearest neighbors is defined as

$$
\mathbb{B}=\left\{\{x, y\}: x, y \in \mathbb{Z}^{2} \text { and }\|x-y\|_{1}=1\right\} .
$$

Given a set $\Gamma \subset \subset \mathbb{Z}^{2}$ we define also

$$
\begin{aligned}
\mathbb{B}_{\Gamma} & =\left\{\{x, y\}: x, y \in \Gamma \text { and }\|x-y\|_{1}=1\right\}, \\
\partial \mathbb{B}_{\Gamma} & =\left\{\{x, y\}: x \in \Gamma, y \notin \Gamma \text { and }\|x-y\|_{1}=1\right\} .
\end{aligned}
$$

Notions from percolation. A chain is a sequence of distinct sites $x_{1}, \ldots, x_{n}$, with the property that for $i=1, \ldots, n-1,\left\|x_{i}-x_{1+1}\right\|_{1}=1$. The sites $x_{1}$ and $x_{n}$ are called the end-points of the chain $x_{1}, \ldots, x_{n}$, and $n$ is its length. A (*)-chain, its endpoints and its length are defined in the same way, but with $\|\cdot\|_{1}$ replaced by $\|\cdot\|_{\infty}$. Informally this means that while chains can only move along bonds of $\mathbb{Z}^{2},(*)$-chains can also move along diagonals. A chain or (*)-chain is said to connect two sets if it has one end-point in each set. A circuit is a chain such that $\left\|x_{1}-x_{n}\right\|_{1}=1$. Similarly a $(*)$-circuit is a $(*)$-chain such that $\left\|x_{1}-x_{n}\right\|_{\infty}=1$.

The Configurations and Observables. At each site in $\mathbb{Z}^{2}$ there is a spin which can take values -1 and +1 . The configurations will therefore be elements of the set 
$\{-1,+1\}^{\mathbb{Z}^{2}}=\Omega$. Given $\sigma \in \Omega$, we write $\sigma(x)$ for the spin at the site $x \in \mathbb{Z}^{2}$. Two configurations are specially relevant, the one with all spins -1 and the one with all spins +1 . We will use the simple notation - and + to denote them. The single spin space, $\{-1,+1\}$ is endowed with the discrete topology and $\Omega$ is endowed with the corresponding product topology. The following definition will be important when we introduce finite systems with boundary conditions later on; given $\Gamma \subset \subset \mathbb{Z}^{2}$ and a configuration $\eta \in \Omega$, we introduce

$$
\Omega_{\Gamma, \eta}=\{\sigma \in \Omega: \sigma(x)=\eta(x) \text { for all } x \notin \Gamma\} .
$$

Real-valued functions with domain in $\Omega$ are called observables. For each observable $f$, we use the notation $\|f\|_{\infty}=\sup _{\eta \in \Omega}|f(\eta)|$. Local observables are those which depend only on the values of finitely many spins, more precisely, $f: \Omega \rightarrow \mathbb{R}$ is a local observable if there exists a set $S \subset \subset \mathbb{Z}^{2}$ such that $f(\sigma)=f(\eta)$ whenever $\sigma(x)=\eta(x)$ for all $x \in S$. The smallest $S$ with this property is called the support of $f$, denoted $\operatorname{supp}(f)$. The topology introduced above on $\Omega$, has the nice feature that it makes the set of local observables be dense in the set of all continuous observables.

For the average spin in a set $\Gamma \subset \subset \mathbb{Z}^{2}$ we will use

$$
X_{\Gamma}(\sigma)=\frac{1}{|\Gamma|} \sum_{x \in \Gamma} \sigma(x)
$$

In $\Omega$ the following partial order is introduced:

$$
\eta \leqq \zeta \text { if } \eta(x) \leqq \zeta(x) \text { for all } x \in \mathbb{Z}^{2} .
$$

The Probability Measures. We endow $\Omega$ also with the Borel $\sigma$-algebra corresponding to the topology introduced above. In this fashion, each probability measure $\mu$ in this space can be identified by the corresponding expected values $\int f d \mu$ of all the local observables $f$. A sequence of probability measures, $\left(\mu_{n}\right)_{n=1,2, \ldots}$, is said to converge weakly to the probability measure $v$ in case

$$
\lim _{n \rightarrow \infty} \int f d \mu_{n}=\int f d v \text { for every continuous observable } f .
$$

The family of probability measures on $\Omega$ will be partially ordered by the following relation: $\mu \leqq v$ if

$$
\int f d \mu \leqq \int f d v \text { for every continuous non-decreasing observable } f .
$$

Because the local observables are dense in the set of continuous observables, we can restrict ourselves to the local ones in (2.1) and (2.2).

The Gibbs Measures. We will consider always the formal Hamiltonian (1.1). In order to give precise definitions, we define, for each set $\Gamma \subset \subset \mathbb{Z}^{2}$ and each boundary condition $\eta \in \Omega$,

$$
H_{\Gamma, \eta, h}(\sigma)=-\frac{1}{2} \sum_{\{x, y\} \in \mathbb{B}_{\Gamma}} \sigma(x) \sigma(y)-\frac{1}{2} \sum_{\substack{\{x, y\} \in \partial \mathbb{B}_{\Gamma} \\ y \notin \Gamma}} \sigma(x) \eta(y)-\frac{h}{2} \sum_{x \in \Gamma} \sigma(x),
$$

where $h \in \mathbb{R}$ is the external field and $\sigma \in \Omega$ is a generic configuration. 
Given $\Gamma \subset \subset \mathbb{Z}^{2}, \eta \in \Omega$, and $E \subset \Omega$, we write

$$
Z_{\Gamma, \eta, T, h}(E)=\sum_{\sigma \in \Omega_{\Gamma, \eta} \cap E} \exp \left(-\beta H_{\Gamma, \eta, h}(\sigma)\right)
$$

where $\beta=1 / T$. We abbreviate $Z_{\Gamma, \eta, T, h}=Z_{\Gamma, \eta, T, h}(\Omega)$.

The Gibbs (probability) measure in $\Gamma$ with boundary condition $\eta$ under external field $h$ and at temperature $T$ is now defined on $\Omega$ as

$$
\mu_{\Gamma, \eta, T, h}(\sigma)= \begin{cases}\frac{\exp \left(-\beta H_{\Gamma, \eta, h}(\sigma)\right)}{Z_{\Gamma, \eta, T, h}}, & \text { if } \sigma \in \Omega_{\Gamma, \eta}, \\ 0, & \text { otherwise. }\end{cases}
$$

The Gibbs measures satisfy the following monotonicity relations to which we will refer as the FKG-Holley inequalities.

If $\eta \leqq \zeta$ and $h_{1} \leqq h_{2}$, then, for each $\Gamma \subset \subset \mathbb{Z}^{2}, \mu_{\Gamma, \eta, T_{1}, h_{1}} \leqq \mu_{\Gamma, \zeta, T_{2}, h_{2}}$.

A Gibbs measure for the infinite system on $\mathbb{Z}^{2}$ is defined now as any probability measure, $\mu$, which satisfies the DLR equations in the sense that for every $\Gamma \subset \subset \mathbb{Z}^{2}$ and $\mu$-almost all $\eta \in \Omega$,

$$
\mu\left(\cdot \mid \Omega_{\Gamma, \eta}\right)=\mu_{\Gamma, \eta, T, h}(\cdot) .
$$

Alternatively and equivalently, Gibbs measures can be defined as elements of the closed convex hull of the set of weak limit points of sequences of the form $\left(\mu_{\Gamma_{i}, \eta_{l}, h}\right)_{i=1,2, \ldots}$, where each $\Gamma_{i}$ is finite and $\Gamma_{i} \rightarrow \mathbb{Z}^{2}$, as $i \rightarrow \infty$, in the sense that $\bigcup_{i=1}^{\infty} \bigcap_{j=i}^{\infty} \Gamma_{j}=\mathbb{Z}^{2}$.

For each value of $T$ and $h, \mu_{\Lambda(l),-, T, h}$ (resp. $\mu_{\Lambda(l),+, T, h}$ ) converges weakly, as $l \rightarrow \infty$, to a probability measure that we will denote by $\mu_{-, T, h}\left(\operatorname{resp} . \mu_{+, T, h}\right)$. If $h \neq 0$ it is known that $\mu_{-, T . h}=\mu_{+, T, h}$, which will then be denoted simply by $\mu_{T, h}$; it is also known that this is the only Gibbs measure for the infinite system in this case. If $h=0$ the same is true if the temperature is larger than or equal to a critical value $T_{c}>0$, and is false for $T<T_{c}$, in which case one says that there is phase coexistence. We use the following abbreviations and names:

$$
\begin{aligned}
& \mu_{-, T, 0}:=\mu_{-, T}=\text { the minus phase }, \\
& \mu_{+, T, 0}:=\mu_{+, T}=\text { the plus phase } .
\end{aligned}
$$

The notation

$$
\mathscr{U}=\left\{(h, T) \in \mathbb{R} \times[0, \infty): h \neq 0 \text { or } T>T_{c}\right\}
$$

will be used to denote the interior of the uniqueness region in the $h \times T$ phasediagram, i.e, the set of all the uniqueness points, except for the critical point $\left(0, T_{c}\right)$.

For the expected value corresponding to a Gibbs measure $\mu \ldots$, in finite or infinite volume, we will use the notation

$$
\langle f\rangle_{\ldots}=\int f d \mu \ldots
$$

where ... stands for arbitrary subscripts. The spontaneous magnetization at temperature $T$ is defined as

$$
m^{*}(T)=\langle\sigma(0)\rangle_{+, T} .
$$

(Here we are using a common and convenient form of abuse of notation: $\sigma(x)$ is being used to denote the observable which associates to each configuration the value 
of the spin at the site $x$ in that configuration. This notation will also be used in other places.) It is known that $m^{*}(T)>0$ if and only if $\mu_{-, T} \neq \mu_{+, T}$.

Surface Tension and Wulff Shape. The direction dependent 0-field surface tension is defined in the following way. First consider on $\mathbb{R}^{2} \times \mathbb{R}^{2}$ the usual inner product $(x, y)=x_{1} y_{1}+x_{2} y_{2}$. Let $\mathbb{S}^{1}=\left\{x \in \mathbb{R}^{2}:\|x\|_{2}=1\right\}$, and for each vector $\mathbf{n} \in \mathbb{S}^{1}$, consider the following configuration, to be used as a boundary condition:

$$
\eta(\mathbf{n})(x)= \begin{cases}+1, & \text { if }(x, \mathbf{n}) \geqq 0, \\ -1, & \text { if }(x, \mathbf{n})<0 .\end{cases}
$$

The surface tension in the direction perpendicular to $\mathbf{n}$ is given by

$$
\tau_{T}(\mathbf{n})=\lim _{l \rightarrow \infty}-\frac{1}{\beta\|y(l)-z(l)\|_{2}} \log \frac{Z_{\Lambda(l), \eta(\mathbf{n}), T, 0}}{Z_{\Lambda(l),+, T, 0}}
$$

where $y(l)$ and $z(l)=-y(l)$ are the points where the straight line $\left\{x \in \mathbb{R}^{2}\right.$ : $(x, n)=0\}$ intersects the boundary of the square $\Lambda(l)$. It is known that for each $T<T_{c}$ the surface tension $\tau_{T}(\cdot)$ is a continuous strictly positive and finite function.

We shall use $\mathscr{D}$ to denote the set of all closed self-avoiding rectifiable curves $\gamma \subset \mathbb{R}^{2}$ that are a boundary of a bounded region, $\gamma=\partial V, V \subset \mathbb{R}^{2}$. Let us recall that a curve is called rectifiable if the supremum of the lengths of polygons, with edges connecting sequentially arbitrary collections of points chosen on the curve, is finite (and equals then the length of the curve), and that a rectifiable curve has a tangent at almost every point. It is easy to verify that a curve $\gamma$ that is the boundary of a convex bounded region belongs to $\mathscr{D}$. We can assign to each curve $\gamma \in \mathscr{D}$ the quantity

$$
\mathscr{W}(\gamma)=\mathscr{W}_{T}(\gamma)=\int_{\gamma} \tau_{T}\left(\mathbf{n}_{s}\right) d s
$$

where $s$ parametrizes the curve $\gamma$ according to Euclidean length measured along this curve, and $\mathbf{n}_{s}$ is the unit outward normal vector to the curve at the point $s \in \gamma$ (i.e. the vector orthogonal to the tangent in the considered point and oriented outward the region bounded by $\gamma$ ). The functional $\mathscr{W}_{T}$ will be called the Wulff functional associated to the zero-field direction-dependent surface tension $\tau_{T}(\cdot)$. Sometimes we will refer to it also as the integrated surface tension.

To every vector $\mathbf{n} \in \mathbb{S}^{1}$ and $\lambda>0$ we assign the half-plane

$$
L_{T, \mathbf{n}, \lambda}=\left\{x \in \mathbb{R}^{2}:(x, \mathbf{n}) \leqq \lambda \tau_{T}(\mathbf{n})\right\} .
$$

Let us consider the intersection

$$
W_{T, \lambda}=\bigcap_{\mathbf{n} \in S^{1}} L_{T, \mathbf{n}, \lambda}
$$

These sets clearly satisfy the scaling relation $W_{T, \lambda}=\lambda W_{T, 1}$. In particular they keep the same shape, as $\lambda$ varies; this shape is called the Wulff shape. The Wulff body of volume 1 is defined as $W_{T}=W_{T, \lambda_{0}}$, where $\lambda_{0}$ is chosen so that its volume is indeed $1 . W_{T}$ is clearly convex and thus its boundary $\partial W_{T} \in \mathscr{D}$. The following is therefore well defined,

$$
w=w(T)=\mathscr{W}_{T}\left(\partial W_{T}\right) .
$$


For each $T<T_{c}$, the boundary of the Wulff body satisfies the following variational principle. For all $\gamma \in \mathscr{D}$ which are boundaries of regions of volume 1,

$$
w(T) \leqq \mathscr{W}_{T}(\gamma)
$$

with equality only in case $\gamma$ is a translation of $\partial W_{T}$.

We will want to consider the Ising model on finite subsets of the lattice $\mathbb{Z}^{2}$ which have an approximate (due to the discreteness of the lattice) Wulff shape. For this purpose we introduce the notation

$$
\Delta(l)=\mathbb{Z}^{2} \cap W_{T, i_{0} l} .
$$

Observe that the volume of this region satisfies $|\Delta(l)|=l^{2}+O(l)$ as $l \rightarrow \infty$.

$A$ Few More Remarks on Notation and Conventions. We will use $C, C_{1}, C_{2}$, etc... to denote positive finite constants, whose precise values are not relevant and may even change from appearance to appearance.

Because of symmetry, several statements that we will make for $h>0$ have obvious analogues for $h<0$, which will not be mentioned.

When we quote results from [Iof2] and other papers, we will sometimes be applying results proven for the system on the box $\Lambda(l)$ to the system on the box $\Delta(l)$. Whenever we do this, we will be implicitly saying that the result holds also in our case, and that the argument needed to prove it would not be different from the one used to prove the quoted result.

The reader should also be aware that some of our conventions are distinct from those in [DKS, Pfi, Iof1 and Iof2]. Three examples are the following. Because the external field that we apply to the Ising model will usually be considered to be positive, we will have to consider the competing boundary condition as -; for this reason we state the large deviation results under no external field also for boundary conditions, rather than for + boundary conditions. In our definition of the surface tension we have a denominator $\beta$ in front of the logarithm of the ratio of partition functions; this factor $\beta$ will appear then in the large deviation rate functions. We define $w(T)$ as the value of the Wulff functional computed on the boundary of $W$, which was normalized to have unit volume; this differs, for instance, from the quantity $\lambda_{F}$ which appears in [Iof2], and which is half the value of the Wulff functional for the boundary of the body that in our notation above is $W_{T, 1}$. It is well known that these quantities are related via $w=2 \sqrt{\lambda_{F}}$, which follows from the fact that the volume of $W_{T, 1}$ is half the Wulff functional of its boundary (this can be obtained by an elementary direct computation, or see (2.7.6) on p. 31 in [DKS]).

\section{Results}

As explained in the introduction the exact second order large deviation estimates for the model without external field and some of the methods used to derive them will be fundamental in our analysis. We start by stating the result in the form in which it is usually presented, then we review the heuristics behind it and state a variant of it which will be of more direct relevance for us. 
Theorem A (Ioffe). Suppose that $T<T_{c}$ and let $m \geqq-m^{*}$ be sufficiently close to $-m^{*}$, then

$$
\lim _{l \rightarrow \infty}-\frac{1}{l} \log \mu_{\Lambda(l),-, T, 0}\left(X_{\Lambda(l)}>m\right)=(\alpha(m))^{1 / 2} \beta w,
$$

with

$$
\alpha(m)=\frac{m+m^{*}}{2 m^{*}} .
$$

The basic picture behind this theorem is that the - boundary condition selects the minus-phase in the bulk, and the best way to produce a value for the average spin in the box higher than the typical value $-m^{*}$ is by the presence of a single droplet of the plus-phase, somewhere, on the background of the minus-phase. The only cost to have it there is the interface, which tends then to adjust itself so as to minimize this cost, expressed by the surface integral of the direction-dependent surface tension. The best possible shape is therefore the Wulff shape, provided it fits into the square box, which is the case if $m$ is close enough to $-m^{*}$. The corresponding probability decays as an exponential of this integrated surface tension multiplied by the inverse temperature $\beta$ (present here because we introduced the factor $1 / \beta$ in the definition of the surface tension). To compute this rate of decay, observe that the volume $v(m)=v$ of the droplet can be computed from

$$
m l^{2}=m^{*} v+\left(-m^{*}\right)\left(l^{2}-v\right) .
$$

Hence

$$
v=\frac{m+m^{*}}{2 m^{*}} l^{2}=\alpha(m) l^{2}
$$

Because the integrated surface tension scales linearly with the linear size of the droplet, we obtain for this integrated surface tension the value $(\alpha(m))^{1 / 2} l w$, which explains the form of the rate function in the theorem above.

The form of the rate function in the situation above, when $m$ is not so close to $-m^{*}$ could in principle also be computed by solving a more complicated variational problem, in which there is an extra constraint (this will be done in [SS]). Nevertheless, for our purposes here, the following remark, that may first sound somewhat artificial, will play a key role: if we replace the box $\Lambda(l)$ with the box $\Delta(l)$, which has the Wulff shape, then this extra difficulty disappears. We state next the result, in the form that we will use it. This result can be proved by combining the techniques used to prove Theorem A above with standard arguments in large deviations theory. The part of the result concerning values of $m$ outside the interval $-m^{*},+m^{*}$, are very well known and go back to the foundations of rigorous statistical mechanics in the 60's (see [Lan, or Oll and FO] for stronger results); to obtain a finite limit in this case, the factor $1 / l$ in front of the logarithm would have to be replaced by $1 / l^{2}$.

Theorem B. Suppose that $T<T_{c}$ and let $-1 \leqq m_{1}<m_{2} \leqq+1$, then

$$
\lim _{l \rightarrow \infty}-\frac{1}{l} \log \mu_{\Delta(l),-, T, 0}\left(X_{\Delta(l)} \in\left(m_{1}, m_{2}\right)\right)=\inf _{m \in\left(m_{1}, m_{2}\right)} \psi(m),
$$

where

$$
\psi(m)= \begin{cases}(\alpha(m))^{1 / 2} \beta w, & \text { if } m \in\left[-m^{*},+m^{*}\right] \\ +\infty, & \text { otherwise }\end{cases}
$$


Next we state our first main result. The fact that the box that we are considering has the Wulff shape may seem strange, but as we will see later on, this theorem has corollaries which concern other shapes, which one may consider as more natural to put the system in, e.g., rectangles. The proofs of parts (a.1) and (b.1) of Theorem 1 will be direct applications of Theorem $B$ and this is the reason we need to consider the system inside of a Wulff-shaped box. It is worth stressing, as we did in the introduction, that Theorem 1 is not in the form of large deviation estimates, but rather a statement on what is likely to happen.

Theorem 1. Suppose that $T<T_{c}$ is fixed and define $B_{0}=B_{0}(T)=w(T) / m^{*}(T)$. Then,

a) If $B<B_{0}$, the following holds:

(1) Given $\varepsilon>0$ there exists $\delta>0$ and $C<\infty$ such that for all $h>0$,

$$
\mu_{\Delta(B / h),-, T, h}\left(X_{\Delta(B / h)} \in\left(-m^{*}-\varepsilon,-m^{*}+\varepsilon\right)\right) \geqq 1-C e^{-\delta / h} .
$$

(2) For each local function $f$,

$$
\langle f\rangle_{\Delta(B / h),-, T, h} \rightarrow\langle f\rangle_{-, T} \text { as } h \searrow 0 .
$$

b) If $B>B_{0}$, the following holds:

(1) Given $\varepsilon>0$ there exists $\delta>0$ and $C<\infty$ such that for all $h>0$,

$$
\mu_{\Delta(B / h),-, T, h}\left(X_{\Delta(B / h)} \in\left(+m^{*}-\varepsilon,+m^{*}+\varepsilon\right)\right) \geqq 1-C e^{-\delta / h} .
$$

(2) Given $\varepsilon>0$ there exists $\delta>0$ and $C<\infty$ such that if we denote by $\mathscr{E}_{h, \varepsilon}$ the event that there is a chain of - spins connecting $\Delta(B(1-\varepsilon) / h)$ to $\partial_{\mathrm{ext}} \Delta(B / h)$, then for all $h>0$,

$$
\mu_{\Delta(B / h),-, T, h}\left(\mathscr{E}_{h, \varepsilon}\right) \leqq C e^{-\delta / h} .
$$

(3) For each local function $f$,

$$
\langle f\rangle_{\Delta(B / h),-, T, h} \rightarrow\langle f\rangle_{+, T} \text { as } h \searrow 0 .
$$

Stronger results will also be presented and discussed below (see (3.3), (3.4) and

The proof of Theorem 1 will be provided in a later section. Here we just want to explain heuristically how it relates to the heuristics behind Theorem B (in the form explained above, after Theorem A was stated). In this heuristics, when $h=0$, one estimates the probability of a given value for the average spin in the box $\Delta(l)(l$ large $)$, by computing the integrated surface tension for a single Wulffshaped droplet of the plus-phase in the background of the minus-phase. In the new problem, the size $l=B / h$ is also large since we consider $h$ as small (the statements in Theorem 1 are only informative for small $h$ ). But the presence of the external field $h$ in the Hamiltonian favors configurations with larger magnetization. For each set of configurations on $\Delta(B / h)$ with a given value of total magnetization, the external field plays no role in modifying their relative probabilities, so that the picture with a single Wulff-shaped droplet should still be the relevant one. From the way the external field enters in the Hamiltonian and the way the Gibbs measures are defined, the effect of the external field in enhancing situations with larger magnetization can be easily accounted for, by multiplying the Gibbs weight (before normalization) corresponding to the magnetization $m$ by a factor $\exp \left(\beta h m^{*} v(m)\right)$, where, as before, 
$v(m)=v$ is the volume of the Wulff droplet when the magnetization is $m$. The reason for this to be the correct expression is that in a region of volume $v(m)$ we are replacing the average magnetization, which would be $-m^{*}$ in the absence of any droplet, by $m^{*}$; the modification is by an amount of $2 m^{*}$, but there is a factor $1 / 2$ in front of $h$ in the way we wrote the Hamiltonian. Recall from the discussion after Theorem A that $v(m)=\left(\left(m+m^{*}\right) /\left(2 m^{*}\right)\right)(B / h)^{2}=\alpha(m)(B / h)^{2}$, so that the logarithm of the weight mentioned above becomes

$$
\beta m^{*} B^{2} \alpha(m) / h \text {. }
$$

Observe that this quantity is of order $1 / h$ just because the volume of the box that we are considering is of order $1 / h^{2}$. The extra weight is tilting the "reference" Gibbs measure under no external field, and we obtain that the probability of seeing the magnetization $m$ is now proportional to the exponential of

$$
\left\{-B(\alpha(m))^{1 / 2} w+m^{*} B^{2} \alpha(m)\right\} \frac{\beta}{h}=-g\left((\alpha(m))^{1 / 2} B\right) \frac{\beta}{h},
$$

where we introduced the function $g:[0, \infty) \rightarrow \mathbb{R}$, given by

$$
g(x)=w x-m^{*} x^{2} .
$$

The behavior of this function is clearly fundamental to understand the typical way the system should look when $h$ is small (recall that $\beta$ is fixed). This behavior is actually very simple: $g(0)=0 ; g(x)$ grows with $x$ up to a critical value $x_{c}$, where it has its absolute maximum; and for $x>x_{c}, g(x)$ decreases as $x$ grows, assuming the value 0 when $x=x_{0}=w / m^{*}$ and becoming negative when $x>x_{0}$. This behavior explains the dichotomy in parts (a) and (b) of Theorem 1: since $\alpha(m)$ runs between 0 and 1 as $m$ runs between $-m^{*}$ and $+m^{*}$, it follows that if $B<B_{0}=x_{0}=w / m^{*}$, then the weight given by the exponential of (3.1) is maximized when $m=-m^{*}$, while if $B>B_{0}=x_{0}=w / m^{*}$, then this weight is maximized when $m=+m^{*}$. One can talk of a "double-well structure," with the deepest well having bottom at $-m^{*}$, or at $+m^{*}$, depending on how $B$ compares with $B_{0}$ (actually if $B<x_{c}$ one is left with a single "well" with bottom at $-m^{*}$, rather than with two "wells," but this is irrelevant for the problems in this paper). To see why part (b.2) of the theorem should be expected, observe that in the case $B>B_{0}$ we expect the typical configurations to correspond to the heuristic picture of a single large droplet of the plus-phase covering essentially the full box $\Delta(B / h)$. A $\left(^{*}\right)$-circuit of + spins is then produced at the border of this droplet, and must be located close to the border of the box; such a $\left(^{*}\right)$-circuit cannot be crossed by any chain of - spins.

Informally speaking, Theorem 1 states that if $B<B_{0}$ we essentially see the system in the minus-phase when $h$ is small, while if $B>B_{0}$ we essentially see the system in the plus-phase when $h$ is small. The precise statements, (a.1) to (b.3), that we made and will prove in detail are not the strongest ones that one can make. We chose the above formulation for the theorem because it seems to us to convey the essentials of the contrast between the two cases (a) and (b), for simplicity of notation and exposition of the proof and because this will be enough to prove Corollary 2 below, on complete analyticity. For completeness we state now some stronger results.

In case $B<B_{0}$ the minus-phase prevails not only in the center of the box, but everywhere, provided that we are not too close to the boundary, where the boundary effect pushes the state even below the minus-phase. To make a precise statement we 
introduce the notation $\theta_{x}(f)$ for the translate by $x \in \mathbb{Z}^{2}$ of the cylindrical function $f$, i.e., $\left(\theta_{x}(f)\right)(\sigma)=f\left(\theta_{x}^{*}(\sigma)\right)$, with $\theta_{x}^{*}(\sigma)(y)=\sigma(y+x)$. The same proof of part (a.2) of Theorem 1 gives the stronger result: If $B<B_{0}$, then for each local function $f$ and function $x(\cdot):(0, \infty) \rightarrow \mathbb{Z}^{2}$, such that

we have

$$
\operatorname{dist}_{2}\left(x(h), \partial_{\text {ext }} \Delta(B / h)\right) \rightarrow \infty \text { as } h \searrow 0,
$$

$$
\left\langle\theta_{x(h)} f\right\rangle_{\Delta(B / h),-, T, h} \rightarrow\langle f\rangle_{-, T} \text { as } h \searrow 0 .
$$

Regarding the case $B>B_{0}$ we can state strengthened versions of parts (b.2) and (b.3) of the theorem. First observe that the complement of the event $\mathscr{E}_{h, \varepsilon}$ in part (b.2) is equivalent to the presence of a $\left({ }^{*}\right)$-circuit of spins + surrounding $\Delta((1-$ $\varepsilon) B / h)$. By partitioning $\left(\mathscr{E}_{h, \varepsilon}\right)^{c}$ according to the outermost such $\left({ }^{*}\right)$-circuit, and using the FKG-Holley inequalities, one can estimate from above the probability of decreasing events with support in $\Delta((1-\varepsilon) B / h)$ by their probabilities with respect to $\mu_{+, T}$. By using Theorem 1 from [CCS] (which states that in the plus-phase the probability of the presence of a $\left({ }^{*}\right)$-chain of - spins connecting two sites decays exponentially with the distance between these sites), and changing $\varepsilon$ into $\varepsilon / 2$ in the statement of part (b.2) of Theorem 1, it follows in this fashion that if $B>B_{0}$, then given $\varepsilon>0$ there exists $\delta>0$ and $C<\infty$ such that if we denote by $\mathscr{E}_{h, \varepsilon}^{*}$ the event that there is a $\left({ }^{*}\right)$-chain of spins - connecting $\Delta(B(1-\varepsilon) / h)$ to $\partial_{\text {ext }} \Delta(B / h)$, then for all $h>0$,

$$
\mu_{\Delta(B / h),-, T, h}\left(\mathscr{E}_{h, \varepsilon}^{*}\right) \leqq C e^{-\delta / h} .
$$

Regarding the extension of part (b.3) of Theorem 1, it is not as strong as that of part (a.2) discussed above. The best result that one can obtain from the techniques used to prove part (b.3) is the following. If $B>B_{0}$, then for each local function $f$ and function $x(\cdot):(0, \infty) \rightarrow \mathbb{Z}^{2}$, such that

$$
\liminf _{h \backslash 0} h \operatorname{dist}_{2}\left(x(h), \partial_{\mathrm{ext}} \Delta(B / h)\right)>0,
$$

we have

$$
\left\langle\theta_{x(h)} f\right\rangle_{\triangle(B / h),-T, h} \rightarrow\langle f\rangle_{+, T} \text { as } h \searrow 0 .
$$

Observe that the condition (3.5) replaces the weaker condition (3.2). While (3.2) cannot obviously be relaxed, if one wants (3.3) to hold, we believe that in order for (3.6) to hold, the critical condition that should replace (3.5) should have $\sqrt{h}$ in place of the factor $h$. The reason is simply that the contour at the border of the droplet of the plus-phase should fluctuate normally over an amplitude of order of the square root of its length. The complete picture should actually be a very rich one, associated with metastability: over a distance of order 1 from the boundary the distribution approaches the minus phase (boundary influence), and only at a distance of order $\sqrt{1 / h}$ there is a change to the plus-phase (magnetic field influence). For each individual realization of the random experiment the interface between the phases should be sharp, at a well defined contour, but the location of this contour fluctuates normally from realization to realization, causing the expected value of local functions to vary quite smoothly with the distance of their support to the boundary of the box.

It is natural to ask how the threshold quantity $B_{0}(T)=w(T) / m^{*}(T)$ behaves as $T \nearrow T_{c}$. It is well known that $m^{*}(T)$ vanishes as $\left(T_{c}-T\right)^{1 / 8}$, while $w(T)$ vanishes as $\left(T_{c}-T\right)^{1}$ (since $\tau_{T}(\mathbf{n})$ has this behavior for each $\mathbf{n} \in \mathbb{S}$; see [AR]). Therefore $B_{0}(T) \sim\left(T_{c}-T\right)^{7 / 8}$ and in particular $B_{0}(T)$ vanishes as $T \nearrow T_{c}$. 
We state next two corollaries to Theorem 1. The first one deals with questions which are similar to those treated in Theorem 1, but this time for square boxes (as the reader can easily guess, analogous results hold for any non-degenerate convex boxes). We present this corollary here as a simple consequence of Theorem 1, but observe that it is not an optimal result. With substantially more work, one can strengthen this result and this will be the content of a companion paper, [SS]. Observe that there is no analogue in this corollary of part (b.1) of Theorem 1; indeed, even for large values of $B$ the sharper results in [SS] will show that close to the corners there will be a fraction of the box where the minus-phase persists.

Corollary 1. For each $T<T_{c}$ there exists positive and finite constants $B_{1}, B_{2}$ and $B_{3}$, which satisfy $B_{1}<B_{2}$ and $B_{3}<B_{2}$ and such that

a) If $B<B_{1}$, the following holds:

(1) Given $\varepsilon>0$ there exists $\delta>0$ and $C<\infty$ such that for all $h>0$,

$$
\mu_{\Lambda(B / h),-, T, h}\left(X_{\Lambda(B / h)} \in\left(-m^{*}-\varepsilon,-m^{*}+\varepsilon\right)\right) \geqq 1-C e^{-\delta / h} .
$$

(2) For each local function $f$,

$$
\langle f\rangle_{\Lambda(B / h),-, T, h} \rightarrow\langle f\rangle_{-, T} \text { as } h \searrow 0 .
$$

b) If $B>B_{2}$, the following holds:

(1) There exists $\delta>0$ and $C<\infty$ such that if we denote by $\mathscr{E}_{h}$ the event that there is a chain of - spins connecting $\Lambda\left(B_{3} / h\right)$ to $\partial_{\mathrm{ext}} \Lambda(B / h)$, then for all $h>0$,

$$
\mu_{\Lambda(B / h),-, T, h}\left(\mathscr{E}_{h}\right) \leqq C e^{-\delta / h} .
$$

(2) For each local function $f$,

$$
\langle f\rangle_{\Lambda(B / h),-, T, h} \rightarrow\langle f\rangle_{+, T} \text { as } h \searrow 0
$$

The proof of the statements above is very simple. Besides Theorem 1, essentially the only other ingredients are the FKG-Holley inequalities. We hint the ideas and leave the details to the reader. For part (a), simply take $B_{1}$ so small that there is a Wulff shape of volume $B^{2}<\left(B_{0}\right)^{2}$, which contains the square of side $B_{1}$. For part (b), take $B_{2}$ so large that a Wulff shape of volume $B^{2}>\left(B_{0}\right)^{2}$ fits inside the square of side $B_{2}$ and choose $B_{3}$ so small that a square of side $B^{\prime}>B_{3}$ fits inside this Wulff shape.

It clearly follows from the proofs sketched above that $B_{1}(T), B_{2}(T)$ and $B_{3}(T)$ can be chosen to vanish as $\left(T_{c}-T\right)^{7 / 8}$ as $T \nearrow T_{c}$.

Corollary 1 , in spite of its non-optimality, as pointed out above, is a strengthening, in the two-dimensional case, of Theorem 1 in [Mar], which states that part (b.1) of this corollary holds at low enough temperatures. On the other hand [Mar] deals with the case of arbitrary dimension $d$, which even at low temperatures is very difficult. Martirosyan's result was reproven in [Sch2], with somewhat simpler methods in arbitrary dimension and with greatly simplified methods in two dimensions; the proofs given in [Sch2] also imply that the constants $B_{1}$ and $B_{2}$ can be taken arbitrarily close to the common value $2 d$, provided the temperature is low enough. This last statement (and the analogue for $B_{3}$ ) can also be obtained, when $d=2$, from the methods in the present paper; observe that the value $2 d=4$ is the limit of $w(T) / m^{*}(T)$ as $T \searrow 0$, when the Wulff shape approaches the shape of a square. 
The main reason this problem was studied in [Sch2] is because of its relation to the metastable behavior of the stochastic Ising models treated in that paper. Actually, as emphasized in Theorem 2 in [Sch2] one can see the behavior described in part (a) of Corollary 1 above as a manifestation of metastability in equilibrium for systems which are not too large and the behavior described in part (b) as the absence of metastability in equilibrium for larger systems. An immediate consequence of the extension of Theorem 2 in [Sch2] up to $T_{c}$ is that also some of the results concerning the dynamical metastability of the stochastic Ising models are extended, with no extra effort up to $T_{c}$; these results are specifically those related to upper bounds on the relaxation time, i.e., parts (ii) of Theorem 1, Theorem 4 and Corollary 1 in that paper. On the other hand it is worth mentioning that further strengthenings of the results in [Sch2] are being obtained and will be the subject of another publication.

The next corollary to Theorem 1, can actually be seen as a corollary to Corollary 1. Its proof is based on Corollary 1 and a more-or-less standard "blocking" (or maybe we should say "renormalization") argument. We will present this proof in Sect. 4.

Corollary 2. For each value of $T \leqq T_{c}$ and $h>0$ there are positive and finite constants $C_{1}$ and $C_{2}$, such that for all $l>0$,

$$
\langle\sigma(0)\rangle_{\Lambda(l),+, T, h}-\langle\sigma(0)\rangle_{\Lambda(l),-, T, h} \leqq C_{1} e^{-C_{2} l} .
$$

Moreover, for $T<T_{c}, C_{2}$ can be chosen independently of $h$.

Combining Corollary 2 with the FKG-Holley inequalities amounts to saying that influence from the boundary in the bulk decays exponentially with the distance from the boundary. Inequality (3.7) is actually equivalent to the notion of "weak mixing" of [MO1, MO2 and MOS] (see part (a) of Theorem 3.3 in [MOS]), defined by saying that for an arbitrary finite subset $\Gamma$ of $\mathbb{Z}^{2}$, if we compare the Gibbs measures with any two boundary conditions, then the distance in total variation between the restrictions of the corresponding Gibbs measures to an arbitrary set $\Gamma^{\prime} \subset \Gamma$ decays exponentially with the Euclidean distance between $\Gamma^{\prime}$ and $\partial_{\mathrm{ext}} \Gamma$. As far as we know, (3.7) had not been proven before in case $T$ is smaller than but close to $T_{c}$ and $h$ is small. On the other hand it had been proved to hold in the following cases:

(1) For large values of $h$, by checking for instance that the single-site Dobrushin uniqueness condition is satisfied in this case.

(2) For low enough $T$ and arbitrary $h>0$; see Corollary 5.1 in [MO1].

(3) For $T>T_{c}$ and arbitrary $h$. This is a particular case of part (i) of Theorem 2 of [Hig].

(4) For $T=T_{c}$ and $h>0$. A proof in this case was available to the authors of [MOS], who nevertheless did not present it in that paper, because it seemed of minor relevance, given the lack of a proof at the time that paper was written in the case now treated in Corollary 2 of the present paper.

Because (3.7) was already known to be true for $T>T_{c}$ and all $h$, we conclude that this property of weak mixing holds for the two-dimensional Ising model in the whole interior $\mathscr{U}$ of the uniqueness region in the $h \times T$ plane.

The fact that we are dealing with a two dimensional lattice system, allows us to draw a much stronger and very relevant conclusion: thanks to the main result in [MOS]-Theorem 1.1 there-we know that the weak mixing condition implies also the condition implicitly called "strong mixing for squares" in that paper, and 
from results in [MO1], and [MO2] several nice consequences follow. To explain the precise form of the conclusions that can be obtained, would require a great deal of notation and definitions. Since this is all already done in [MOS], we will limit ourselves below to giving a brief and somewhat sketchy description of the results and their connections to previous work.

The notion of "strong mixing for cubes" is a modification, in an important fashion, of that of "complete analyticity." "Complete analyticity" was introduced in [DS2] and [DS3] for lattice systems which are much more general than the Ising model that we are considering, and can be defined (in arbitrary dimension) by saying that for an arbitrary finite subset $\Gamma$ of $\mathbb{Z}^{d}$, if we compare the Gibbs measures with two boundary conditions which only differ at a single site $y$, then the distance in total variation between the restrictions of the corresponding Gibbs measures to an arbitrary set $\Gamma^{\prime} \subset \Gamma$ decays exponentially with the Euclidean distance from $y$ to $\Gamma^{\prime}$. Observing that $\Gamma^{\prime}$ could be close to the boundary of $\Gamma$, but far from $y$, one sees why this condition is stronger than the notion of "weak mixing," as informally described above. Indeed, in dimensions higher than 2 it is known (see [Sh1]) that "weak mixing" can hold while "complete analyticity" fails. In the papers [DS2 and DS3] complete analyticity was shown to be equivalent to several properties of the Gibbs measures restricted to finite subsets of the lattice. All these properties were in the form of some estimates which were uniform over all the finite subsets of the lattice. More recently, Stroock and Zegarlinski showed in [SZ] that complete analyticity is also equivalent to some statements about the corresponding Glaubertype dynamics (i.e., reversible spin flip dynamics) and their corresponding Dirichlet forms - including logarithmic Sobolev inequalities, and exponential convergence to equilibrium. Again all the statements were uniform over all finite subsets of the lattice.

It is natural to ask in the case of concrete models, like the Ising model that we are considering, for which values of the parameters one has all these properties. It was realized that the notion of complete analyticity as originally defined, uniform over all finite subsets of the lattice, is actually too strong to hold in certain cases in which one still expects the system to have a very decent behavior. An explicit twodimensional counterexample, due to one of us, was described in [MOS1], where the Hamiltonian considered is just slightly more complicated than the one treated in this paper. We will not reproduce any counterexample here, but simply stress the fact that if one considers arbitrary subsets of $\mathbb{Z}^{2}$, then pathologies are not unexpected, since the subset may have a boundary which is comparable in size to the set itself. From the point of view of the physics involved in such problems, one would be satisfied with a condition of complete analyticity restricted to "reasonable" subsets of the lattice, including rectangles, say. A project of this type was carried out by Martinelli and Olivieri in [MO1, MO2] and related results appeared also in [LY]. In these papers results similar to those of Stroock and Zegarlinski were proven, in the form of equivalences between statements of complete analyticity, properties of reversible spin-flip dynamics and logarithmic Sobolev inequalities, uniformly only over certain subsets of the lattice, including all cubes.

In dimension 3 or higher, the counterexamples in [Shl] show that even if we restrict the definition of complete analyticity, admitting only sets $\Gamma \subset \subset \mathbb{Z}^{d}$ which are cubes, it still does not follow from weak mixing. On the other hand, in [MOS] it was shown (as already mentioned above) that in two dimensions this restricted form of complete analyticity does follow from weak mixing. Therefore we can state now the following theorem. 
Theorem 2. For each $(h, T) \in \mathscr{U}$ the equivalent statements (i)-(vi) of Theorem 3.2 of $[M O S]$ hold.

We refer the reader to [MOS] for the precise statements and the various necessary definitions, but we can summarize here the six statements as follows:

(i) - Weak mixing.

(ii) - Restricted complete analyticity, in the sense that the set $\Gamma$ in the definition that we reviewed above is restricted to the squares.

(iii) - Exponential convergence to equilibrium of the associated Glauber dynamics uniformly over the squares of the lattice, uniformly over boundary conditions and over initial conditions.

(iv) - Positive lower bound for the spectral gap of the generator of the associated Glauber dynamics, uniform over the squares of the lattice with arbitrary boundary conditions.

(v) - Finite upper bound for the logarithmic Sobolev constant of the generator of the associated Glauber dynamics, uniform over the squares of the lattice with arbitrary boundary conditions.

(vi) - A constructive condition for uniqueness of the Gibbs measure in infinite volume which was introduced by Dobrushin and one of us in [DS1] is satisfied.

Moreover the equivalent nice properties obtained are valid not only for squares, as explicitly stated in [MOS] and above, but for a much larger class of subsets of the lattice, including the sets which are disjoint unions of translates of a large enough square and also all rectangles.

We comment now on the statement in the last line of Corollary 2. It may seem surprising at first sight that the constant $C_{2}$ there depends on $T$ but not on $h>0$, since this means that it does not vanish when one approaches the transition line. A little thought, though, indicates that this should actually be expected. One can think of the optimal value of $C_{2}$ there as the inverse of a "correlation length," and correlation lengths are not expected to diverge as one approaches a first order transition, which is the case here.

\section{Proofs of Theorem 1 and Corollary 2}

Proof of part (a.1) of Theorem 1. Given $-1 \leqq m_{1}<m_{2} \leqq 1$, we estimate $\mu_{\Delta(B / h),-, T h}\left(X_{\Delta(B / h)} \in\left(m_{1}, m_{2}\right)\right)$ as follows, where $\delta_{1}>0$ is arbitrary,

$$
\begin{aligned}
& \mu_{\Delta(B / h),-, T, h}\left(X_{\Delta(B / h)} \in\left(m_{1}, m_{2}\right)\right)=\frac{Z_{\Delta(B / h),-, T, h}\left(X_{\Delta(B / h)} \in\left(m_{1}, m_{2}\right)\right)}{Z_{\Delta(B / h),-, T, h}} \\
& \quad \leqq \frac{Z_{\Delta(B / h),-, T, h}\left(X_{\Delta(B / h)} \in\left(m_{1}, m_{2}\right)\right)}{Z_{\Delta(B / h),-, T, h}\left(X_{\Delta(B / h)} \in\left(-m^{*}-\delta_{1},-m^{*}+\delta_{1}\right)\right)} \\
& \leqq \frac{Z_{\Delta(B / h),-, T, 0}\left(X_{\Delta(B / h)} \in\left(m_{1}, m_{2}\right)\right)}{Z_{\Delta(B / h),-, T, 0}\left(X_{\Delta(B / h)} \in\left(-m^{*}-\delta_{1},-m^{*}+\delta_{1}\right)\right)} \frac{e^{\beta(h / 2) m_{2}|\Delta(B / h)|}}{e^{\beta(h / 2)\left(-m^{*}-\delta_{1}\right)|\Delta(B / h)|}} \\
& \quad=\frac{\mu_{\Delta(B / h),-, T, 0}\left(X_{\Delta(B / h)} \in\left(m_{1}, m_{2}\right)\right)}{\mu_{\Delta(B / h),-, T, 0}\left(X_{\Delta(B / h)} \in\left(-m^{*}-\delta_{1},-m^{*}+\delta_{1}\right)\right)} \frac{e^{\beta(h / 2) m_{2}|\Delta(B / h)|}}{e^{\beta(h / 2)\left(-m^{*}-\delta_{1}\right)|\Delta(B / h)|}} .
\end{aligned}
$$


From Theorem B, the fact that $|\Delta(B / h)|=(B / h)^{2}+O(1 / h)$, and the arbitrariness of $\delta_{1}$, it follows that

$$
\begin{aligned}
\liminf _{h \searrow 0}-h \log \mu_{\Delta(B / h),-, T, h}\left(X_{\Delta(B / h)} \in\left(m_{1}, m_{2}\right)\right) \\
\geqq\left(\inf _{m \in\left(m_{1}, m_{2}\right)} \psi(m)\right) B-\beta \frac{m_{2}+m^{*}}{2} B^{2} .
\end{aligned}
$$

For each $m \in[-1,+1]$ we can take an interval $\left(m_{1}, m_{2}\right)$ centered at $m$, with the property that the right-hand side of $(4.1)$ is as close as desired to $\psi(m) B-$ $\beta\left(\left(m+m^{*}\right) / 2\right) B^{2}$. This is the case even if $m \notin\left[-m^{*},+m^{*}\right]$, in which case this expression is identical to $+\infty$. On the other hand, if $m \in\left[-m^{*},+m^{*}\right]$, then this expression is identical to $\beta\left\{w(\alpha(m))^{1 / 2} B-\left(\left(m+m^{*}\right) / 2\right) B^{2}\right\}=\beta\left\{w(\alpha(m))^{1 / 2} B-\right.$ $\left.m^{*} \alpha(m) B^{2}\right\}=\beta g\left((\alpha(m))^{1 / 2} B\right)$. Because $B<B_{0}(T)=w / m^{*}$ and $\alpha(m) \in(0,1]$ when $m \in\left(-m^{*},+m^{*}\right]$, it follows that $g\left((\alpha(m))^{1 / 2} B\right)>0$ for $m \in\left(-m^{*},+m^{*}\right]$. We can conclude that for each $m \in\left[-1,-m^{*}-\varepsilon\right] \cup\left[-m^{*}+\varepsilon,+1\right]$ we can take the interval $\left(m_{1}, m_{2}\right)$ centered at $m$, small enough, so that the right-hand side in (4.1) is positive. By compactness, we can now select a finite set $\mathscr{I}$ of such intervals $\left(m_{1}, m_{2}\right)$ which covers the set $\left[-1,-m^{*}-\varepsilon\right] \cup\left[-m^{*}+\varepsilon,+1\right]$. Part (a.1) of the theorem clearly follows now from (4.1) with any positive $\delta$ smaller than

$$
\min _{\left(m_{1}, m_{2}\right) \in \mathscr{I}}\left\{\left(\inf _{m \in\left(m_{1}, m_{2}\right)} \psi(m)\right) B-\beta\left(\left(m_{2}+m^{*}\right) / 2\right) B^{2}\right\} .
$$

Proof of part (a.2) of Theorem 1. We will first consider the particular case of the observables defined by $f(\sigma)=\sigma\left(x_{0}\right)$, for some $x_{0} \in \mathbb{Z}^{2}$. A direct application of the fact that the Gibbs measures are increasing with the external field $h$ gives

$$
\liminf _{h \backslash 0}\left\langle\sigma\left(x_{0}\right)\right\rangle_{\Delta(B / h),-, T, h} \geqq \liminf _{h \backslash 0}\left\langle\sigma\left(x_{0}\right)\right\rangle_{\Delta(B / h),-, T, 0}=\left\langle\sigma\left(x_{0}\right)\right\rangle_{-, T}=-m^{*} .
$$

So it remains to show that

$$
\underset{h \searrow 0}{\lim \sup }\left\langle\sigma\left(x_{0}\right)\right\rangle_{\Delta(B / h),-, T, h} \leqq-m^{*}
$$

We will argue by contradiction, and so we suppose that

$$
\underset{h \backslash 0}{\lim \sup }\left\langle\sigma\left(x_{0}\right)\right\rangle_{\Delta(B / h),-, T, h}=m>-m^{*} .
$$

Since $B<B_{0}$ we can take $B^{\prime} \in\left(B, B_{0}\right)$. Then from part (a.1) of the theorem and the Dominated Convergence Theorem, we know that

$$
\lim _{h \backslash 0}\left\langle X_{\Delta\left(B^{\prime} / h\right)}\right\rangle_{\Delta\left(B^{\prime} / h\right),-, T, h}=-m^{*} .
$$

By elementary geometric arguments, it is clear that there is $\delta>0$, small enough, so that for all $x \in \Lambda(\delta / h)$, the translate by $x$ of $\Delta(B / h)$ is contained in $\Delta\left(B^{\prime} / h\right)$. By translation invariance of the Hamiltonian and the FKG-Holley inequalities, it follows that for all $x \in x_{0}+\Lambda(\delta / h)$,

$$
\langle\sigma(x)\rangle_{\triangle\left(B^{\prime} / h\right),-, T, h} \geqq\left\langle\sigma\left(x_{0}\right)\right\rangle_{\Delta(B / h),-, T, h} .
$$


Look now at $\Delta\left(B^{\prime} / h\right) \backslash\left[x_{0}+\Lambda(\delta / h)\right]=\Gamma(h)$, and observe that if we define $\widetilde{\Gamma}(h)=\left\{y \in \Gamma(h): \operatorname{dist}_{\infty}\left(y, \partial_{\text {ext }} \Delta\left(B^{\prime} / h\right)\right)>\sqrt{1 / h}\right\}$, then using the FKG-Holley inequalities,

$$
\begin{aligned}
\liminf _{h \backslash 0} & \left\langle X_{\Gamma(h)}\right\rangle_{\Delta\left(B^{\prime} / h\right),-, T, h} \geqq \liminf _{h \backslash 0}\left\langle X_{\Gamma(h)}\right\rangle_{\Delta\left(B^{\prime} / h\right),-, T, 0} \\
& =\liminf _{h \searrow 0}\left\langle X_{\widetilde{\Gamma}(h)}\right\rangle_{\Delta\left(B^{\prime} / h\right),-, T, 0} \geqq \lim _{h \backslash 0}\langle\sigma(0)\rangle_{\Lambda}(\sqrt{1 / h}),-, T, 0 \\
& =-m^{*} .
\end{aligned}
$$

But together (4.3), (4.5) and (4.6) imply that

$$
\limsup _{h \searrow 0}\left\langle X_{\Delta\left(B^{\prime} / h\right)}\right\rangle_{\Delta\left(B^{\prime} / h\right),-, T, h}>-m^{*},
$$

in contradiction with (4.4), completing the proof for this choice of $f$.

To show that (4.2) must hold for arbitrary $f$, one could invoke the fact that it is enough to prove it for increasing $f$ and for those one could imitate the complete argument above, by first proving the analogue of part (a.1) with $X_{\Delta(B / h)}$ replaced with the similar average over $\Delta(B / h)$ of translates of $f$. Alternatively, we will use a standard type of coupling to show that the result for arbitrary $f$ follows from the particular case treated above.

Because $\mu_{\Delta(B / h),-, T, 0} \leqq \mu_{\Delta(B / h),-, T, h}$, there exists a probability measure $\mathbb{P}$ on $\Omega \times \Omega$, with the properties that

(1) $\mathbb{P}(\mathscr{A}, \Omega)=\mu_{\Delta(B / h),-, T, 0}(\mathscr{A})$ for each event $\mathscr{A} \subset \Omega$.

(2) $\mathbb{P}(\Omega, \mathscr{A})=\mu_{\Delta(B / h),-T, h}(\mathscr{A})$ for each event $\mathscr{A} \subset \Omega$.

(3) $\mathbb{P}(\{(\eta, \zeta): \eta \leqq \zeta\})=1$.

Using this coupling we can write

$$
\begin{gathered}
\left|\langle f\rangle_{\Delta(B / h),-, T, h}-\langle f\rangle_{\Delta(B / h),-, T, 0}\right|=\left|\int f(\zeta) d \mathbb{P}(\eta, \zeta)-\int f(\eta) d \mathbb{P}(\eta, \zeta)\right| \\
\leqq 2\|f\|_{\infty} \mathbb{P}(\{(\eta, \zeta): \eta(x) \neq \zeta(x) \text { for some } x \in \operatorname{supp}(f)\}) .
\end{gathered}
$$

But

$$
\lim _{h \searrow 0}\langle f\rangle_{\Delta(B / h),-, T, 0}=\langle f\rangle_{-, T} .
$$

And

$$
\begin{aligned}
\mathbb{P}(\{(\eta, \zeta): \eta(x) & \neq \zeta(x) \text { for some } x \in \operatorname{supp}(f)\}) \\
& \leqq \sum_{x \in \operatorname{supp}(f)} \mathbb{P}(\{(\eta, \zeta): \eta(x)<\zeta(x)\}) \\
& =\frac{1}{2} \sum_{x \in \operatorname{supp}(f)} \int(\zeta(x)-\eta(x)) d \mathbb{P}(\eta, \zeta) \\
& =\frac{1}{2} \sum_{x \in \operatorname{supp}(f)}\left(\langle\sigma(x)\rangle_{\Delta(B / h),-, T, h}-\langle\sigma(x)\rangle_{\Delta(B / h),-, T, 0}\right),
\end{aligned}
$$

which we already know goes to 0 as $h \searrow 0$. 
Proof of part (b.1) of Theorem 1. The proof of part (b.1) of the theorem is similar to that of part (a.1). We start by estimating, $\mu_{\Delta(B / h),-T, h}\left(X_{\Delta(B / h)} \in\left(m_{1}, m_{2}\right)\right)$ for each $-1 \leqq m_{1}<m_{2} \leqq 1$, in a way which is close, but not identical to the one used for part (a.1), with $\delta_{1}>0$ again arbitrary,

$$
\begin{aligned}
& \mu_{\Delta(B / h),-T, h}\left(X_{\Delta(B / h)} \in\left(m_{1}, m_{2}\right)\right)=\frac{Z_{\Delta(B / h),-, T, h}\left(X_{\Delta(B / h)} \in\left(m_{1}, m_{2}\right)\right)}{Z_{\Delta(B / h),-, T, h}} \\
& \leqq \frac{Z_{\Delta(B / h),-, T, h}\left(X_{\Delta(B / h)} \in\left(m_{1}, m_{2}\right)\right)}{Z_{\Delta(B / h),-, T, h}\left(X_{\Delta(B / h)} \in\left(+m^{*}-\delta_{1},+m^{*}+\delta_{1}\right)\right)} \\
& \leqq \frac{Z_{\Delta(B / h),-, T, 0}\left(X_{\Delta(B / h)} \in\left(m_{1}, m_{2}\right)\right)}{Z_{\Delta(B / h),-, T, 0}\left(X_{\Delta(B / h)} \in\left(+m^{*}-\delta_{1},+m^{*}+\delta_{1}\right)\right)} \frac{e^{\beta(h / 2) m_{2}|\Delta(B / h)|}}{e^{\beta(h / 2)\left(m^{*}-\delta_{1}\right)|\Delta(B / h)|}} \\
& =\frac{\mu_{\Delta(B / h),-, T, 0}\left(X_{\Delta(B / h)} \in\left(m_{1}, m_{2}\right)\right)}{\mu_{\Delta(B / h),-, T, 0}\left(X_{\Delta(B / h)} \in\left(+m^{*}-\delta_{1},+m^{*}+\delta_{1}\right)\right)} \frac{e^{\beta(h / 2) m_{2}|\Delta(B / h)|}}{e^{\beta(h / 2)\left(m^{*}-\delta_{1}\right)|\Delta(B / h)|}}
\end{aligned}
$$

From Theorem B, the fact that $|\Delta(B / h)|=(B / h)^{2}+O(1 / h)$, and the arbitrariness of $\delta_{1}$, it follows that

$$
\begin{aligned}
\liminf _{h \backslash 0}-h \log \mu_{\Delta(B / h),-, T, h}\left(X_{\Delta(B / h)} \in\left(m_{1}, m_{2}\right)\right) \\
\quad \geqq\left(\inf _{m \in\left(m_{1}, m_{2}\right)} \psi(m)\right) B-\psi\left(m^{*}\right) B-\beta \frac{m_{2}-m^{*}}{2} B^{2} .
\end{aligned}
$$

For each $m \in[-1,+1]$ we can take an interval $\left(m_{1}, m_{2}\right)$ centered at $m$, with the property that the right-hand side of (4.7) is as close as desired to $[\psi(m) B-\beta((m+$ $\left.\left.\left.m^{*}\right) / 2\right) B^{2}\right]-\left[\psi\left(m^{*}\right) B-\beta m^{*} B^{2}\right]$. This is the case even if $m \notin\left[-m^{*},+m^{*}\right]$, in which case this expression is identical to $+\infty$. On the other hand, if $m \in\left[-m^{*},+m^{*}\right]$, then this expression is identical to

$$
\begin{aligned}
& {\left[\beta\left\{w(\alpha(m))^{1 / 2} B-\left(\left(m+m^{*}\right) / 2\right) B^{2}\right\}\right]-\left[\beta\left\{w\left(\alpha\left(m^{*}\right)\right)^{1 / 2} B-m^{*} B^{2}\right\}\right]} \\
& \quad=\beta\left\{\left[w(\alpha(m))^{1 / 2} B-m^{*} \alpha(m) B^{2}\right]-\left[w\left(\alpha\left(m^{*}\right)\right)^{1 / 2} B-m^{*} \alpha\left(m^{*}\right) B^{2}\right]\right\} \\
& \quad=\beta\left\{g\left((\alpha(m))^{1 / 2} B\right)-g\left(\left(\alpha\left(m^{*}\right)\right)^{1 / 2} B\right)\right\} .
\end{aligned}
$$

Because $B>B_{0}(T)=w / m^{*}$ and $\alpha(m) \in[0,1)$ when $m \in\left[-m^{*},+m^{*}\right)$, it follows that $g\left(\left(\alpha(m)^{1 / 2} B\right)>g\left(\left(\alpha\left(m^{*}\right)\right)^{1 / 2} B\right)\right.$ for $m \in\left[-m^{*},+m^{*}\right)$. We can conclude that for each $m \in\left[-1,+m^{*}-\varepsilon\right] \cup\left[+m^{*}+\varepsilon,+1\right]$ we can take the interval $\left(m_{1}, m_{2}\right)$ centered at $m$, small enough, so that the right-hand side in (4.7) is positive. By compactness, we can now select a finite set $\mathscr{I}$ of such intervals $\left(m_{1}, m_{2}\right)$ which covers the set $\left[-1,+m^{*}-\varepsilon\right] \cup\left[+m^{*}+\varepsilon,+1\right]$. Part (b.1) of the theorem clearly follows now from (4.7) with any positive $\delta$ smaller than

$$
\min _{\left(m_{1}, m_{2}\right) \in \mathscr{I}}\left\{\left(\inf _{m \in\left(m_{1}, m_{2}\right)} \psi(m)\right) B-\psi\left(m^{*}\right) B-\beta\left(\left(m_{2}-m^{*}\right) / 2\right) B^{2}\right\}
$$


Proof of part (b.2) of Theorem 1. For this proof we will need, besides of the results from [Iof2] also techniques from that paper as well as from [DKS]. Motivated by part (b.1), proved above we write:

$$
\begin{aligned}
\mu_{\Delta(B / h),-, T, h}\left(\mathscr{E}_{h, \varepsilon}\right) \leqq & \mu_{\Delta(B / h),-, T, h}\left(\mathscr{E}_{h, \varepsilon}, X_{\Delta(B / h)} \in\left(+m^{*}-\delta_{1},+m^{*}+\delta_{1}\right)\right) \\
& +\mu_{\Delta(B / h),-, T, h}\left(X_{\Delta(B / h)} \notin\left(+m^{*}-\delta_{1},+m^{*}+\delta_{1}\right)\right)
\end{aligned}
$$

The first of these two terms will be estimated by writing

$$
\begin{aligned}
\mu_{\Delta(B / h),-T, h}\left(\mathscr{E}_{h, \varepsilon}, X_{\Delta(B / h)} \in\left(+m^{*}-\delta_{1},+m^{*}+\delta_{1}\right)\right) \\
=\frac{Z_{\Delta(B / h),-, T, h}\left(\mathscr{E}_{h, \varepsilon}, X_{\Delta(B / h)} \in\left(+m^{*}-\delta_{1},+m^{*}+\delta_{1}\right)\right)}{Z_{\Delta(B / h),-, T, h}} \\
\leqq \frac{Z_{\Delta(B / h),-, T, h}\left(\mathscr{E}_{h, \varepsilon}, X_{\Delta(B / h)} \in\left(+m^{*}-\delta_{1},+m^{*}+\delta_{1}\right)\right)}{Z_{\Delta(B / h),-, T, h}\left(X_{\Delta(B / h)} \in\left(+m^{*}-\delta_{1},+m^{*}+\delta_{1}\right)\right)} \\
\leqq \frac{Z_{\Delta(B / h),-, T, 0}\left(\mathscr{E}_{h, \varepsilon}, X_{\Delta(B / h)} \in\left(+m^{*}-\delta_{1},+m^{*}+\delta_{1}\right)\right)}{Z_{\Delta(B / h),-, T, 0}\left(X_{\Delta(B / h)} \in\left(+m^{*}-\delta_{1},+m^{*}+\delta_{1}\right)\right)} e^{|\Delta(B / h)| \beta h \delta_{1}} .
\end{aligned}
$$

Combining the two previous inequalities with part (b.1) of the theorem yields

$$
\begin{aligned}
\mu_{\Delta(B / h),-, T, h}\left(\mathscr{E}_{h, \varepsilon}\right) \leqq & \frac{\mu_{\Delta(B / h),-, T, 0}\left(\mathscr{E}_{h, \varepsilon}, X_{\Delta(B / h)} \in\left(+m^{*}-\delta_{1},+m^{*}+\delta_{1}\right)\right)}{\mu_{\Delta(B / h),-, T, 0}\left(X_{\Delta(B / h)} \in\left(+m^{*}-\delta_{1},+m^{*}+\delta_{1}\right)\right)} e^{|\Delta(B / h)| \beta h \delta_{1}} \\
& +C_{1} e^{-C_{2} / h}
\end{aligned}
$$

where $C_{1}$ and $C_{2}$ depend on the choice of $\delta_{1}$, but are positive and finite for each $\delta_{1}>0$.

The denominator of the ratio of probabilities in (4.8) can be estimated using Theorem B by

$$
\begin{aligned}
\lim _{h \searrow 0}-h \log \mu_{\Delta(B / h),-, T, 0}\left(X_{\Delta(B / h)} \in\left(m^{*}-\delta_{1}, m^{*}+\delta_{1}\right)\right) & =B\left(\alpha\left(m^{*}-\delta_{1}\right)\right)^{1 / 2} \beta w \\
& =B\left(1-\delta_{1} /\left(2 m^{*}\right)\right)^{1 / 2} \beta w .
\end{aligned}
$$

To estimate the numerator of this ratio, we will split the corresponding event into three parts. In order to define this splitting, we will need now to recall several notions from [DKS and Iof2]. We suppose that the reader is familiar with the notion of contours on the dual lattice $\mathbb{Z}^{2}+(1 / 2,1 / 2)$, which separate spins -1 from +1 , and we adopt here the splitting rules used in [DKS] (see Sect. 3.1 there), which allow one to take the contours as self-avoiding closed curves (when the boundary conditions are -, which is our case). We will denote by $|\Gamma|$ the length of the contour $\Gamma$ and by $V(\Gamma)$ the number of spins that it surrounds, which we call the volume of $\Gamma$. In what follows $b$ is a fixed but arbitrary number in $(0,1 / 4)$. A contour $\Gamma$ will be said to be large if $V(\Gamma)>(1 / h)^{2 b}$, otherwise $\Gamma$ will be said to be small. A basic tool to deal with the large contours is the notion of "skeleton." To introduce it we choose a second arbitrary number $v \in(0, b)$. Given now a large contour $\Gamma$ one can associate to it, in an algorithmic way, a sequence of sites, $\left(x_{1}, \ldots, x_{J}\right)$ of the 
dual lattice $\mathbb{Z}^{2}+(1 / 2,1 / 2)$. We think of the sites $x_{1}, \ldots, x_{J}$ as the ordered vertices of a closed polygonal curve, with possible self-intersections (see Fig. 5.3 on p. 166 in [DKS]); we will denote this curve by $\gamma$ in what follows and call it the skeleton of $\Gamma$. For the construction of $\gamma$, given $\Gamma$, the reader is referred to Chapter 5 of [DKS]; here we will limit ourselves to reviewing some of the basic properties that we can guarantee the skeleton to have:

(1) $x_{i} \in \Gamma$ for each $i$.

(2) The length of each edge of $\gamma$ is bounded between $C_{3}(1 / h)^{v}$ and $C_{4}(1 / h)^{v}$, where $0<C_{3}<C_{4}<\infty$ are fixed appropriate constants.

(3) The Hausdorff distance between $\Gamma$ and $\gamma$ satisfies

$$
\rho_{H}(\Gamma, \gamma) \leqq(1 / h)^{v} .
$$

The length, $|\gamma|$ of a skeleton $\gamma$ is defined as the sum of the Euclidean lengths of its edges. To each skeleton $\gamma$ we associate its Wulff functional, $\mathscr{W}(\gamma)$, defined by summing over the edges of $\gamma$ the product of the Euclidean length of each edge by the surface tension in the direction defined by the edge, i.e., $\tau_{T}(\mathbf{n})$, with $\mathbf{n}$ perpendicular to the edge. Observe that from the fact that the surface tension $\tau_{T}(\mathbf{n})$ is bounded away from 0 and $\infty$ uniformly in $\mathbf{n}$,

$$
C_{5} \mathscr{W}(\gamma) \leqq|\gamma| \leqq C_{6} \mathscr{W}(\gamma)
$$

As usual, a contour is called an external contour if it is not enclosed by any other contour. To each configuration $\sigma \in \Omega_{\Delta(B / h),-}$ we can associate the collection $G=\left\{\Gamma_{1}, \ldots, \Gamma_{n}\right\}$ of external large contours. To this collection we can associate the collection $S=\left\{\gamma_{1}, \ldots, \gamma_{n}\right\}$ of their skeletons. The Wulff functional associated to the configuration $\sigma$ is then defined as

$$
\mathscr{W}(S)=\sum_{i=1}^{n} \mathscr{W}\left(\gamma_{i}\right)
$$

with the convention that $\mathscr{W}(\emptyset)=0$. Next we want to consider the volume surrounded by the external large contours $\Gamma_{1}, \ldots, \Gamma_{n}$ and say that it has to be close to the volume surrounded by the collection of skeletons $\gamma_{1}, \ldots, \gamma_{n}$. A difficulty lies in the fact that while the volume surrounded by the contours is easily defined as

$$
V(G)=\sum_{i=1}^{n} V\left(\Gamma_{i}\right)
$$

the fact that the skeletons can self-intersect and also intersect with each other, makes the notion of the volume that they surround more delicate. Fortunately the notion of "phase volume," as defined in Sect. 2.10 of [DKS], solves our difficulty. This definition is as follows (a look at Fig. 2.5 on p. 37 of [DKS] will probably lead the reader to guess correctly the definition). The set $\mathbb{R}^{2} \backslash \cup \gamma_{i}$ splits up into a collection of connected components $Q_{\alpha}$ with exactly one unbounded component among them. A component $Q_{\alpha}$ will be called a minus-component if any path that connects its interior points with points of the unbounded component and intersects the curves from $S$ in a finite number of points, intersects them in an odd number of points (counted with multiplicities). The phase volume of $S$, denoted by $\hat{V}(S)$, is defined as the joint volume of all the minus-components.

Motivated by $(4.10)$, we want to show that $V(G)$ and $\hat{V}(S)$ have also to be relatively close. If we remove from $\mathbb{R}^{2}$ all the points which are at a distance not 
larger than $(1 / h)^{y}$ from $\cup \Gamma_{i}$, then the remaining set also splits up into connected components with exactly one unbounded component among them. It is easy to see that all the bounded components are subsets of minus-components in the splitting produced by $S$, while the unbounded component is a subset of the unbounded component in the splitting produced by $S$. It is also clear that the bounded components in this splitting are inside contours of $G$, while the unbounded component in this splitting is completely outside the contours of $G$. Hence

$$
|V(G)-\hat{V}(S)| \leqq C_{7}\left(\sum_{i=1}^{n}\left|\Gamma_{i}\right|\right)(1 / h)^{2 v}
$$

We are now ready to define the following exhaustive set of events:

(1) $\mathscr{F}_{1}$ will be the event that $\mathscr{W}(S)>\left(1+\delta_{2}\right) \beta w B / h$, where the constant $\delta_{2}$ will be chosen later.

(2) $\mathscr{F}_{2}$ will be the event that $\mathscr{W}(S) \leqq\left(1+\delta_{2}\right) \beta w B / h$ and $\hat{V}(S)<\left(1-\delta_{1} / m^{*}\right)$ $(B / h)^{2}$.

(3) $\mathscr{F}_{3}$ will be the event that $\mathscr{W}(S) \leqq\left(1+\delta_{2}\right) \beta w B / h$ and $\hat{V}(S) \geqq\left(1-\delta_{1} / m^{*}\right)$ $(B / h)^{2}$.

The following inequality is a particular case of (4.3) in [Iof2], which is based on ideas in [Pfi] (in its derivation properties (1) and (2) of the skeletons are used),

$$
\liminf _{h \searrow 0}-h \log \mu_{\Delta(B / h),-, T, 0}\left(\mathscr{F}_{1}\right) \geqq\left(1+\delta_{2}\right) \beta w B .
$$

Combining this inequality with (4.9), we obtain

$$
\begin{aligned}
& \liminf _{h \backslash 0}-h \log \frac{\mu_{\Delta(B / h),-, T, 0}\left(\mathscr{E}_{h, \varepsilon}, X_{\Delta(B / h)} \in\left(m^{*}-\delta_{1}, m^{*}+\delta_{1}\right), \mathscr{F}_{1}\right)}{\mu_{\Delta(B / h),-, T, 0}\left(X_{\Delta(B / h)} \in\left(m^{*}-\delta_{1}, m^{*}+\delta_{1}\right)\right)} e^{|\Delta(B / h)| h \delta_{1}} \\
& \geqq\left[\left(1+\delta_{2}\right)-\left(1-\delta_{1} /\left(2 m^{*}\right)\right)^{1 / 2}\right] \beta w B-\beta B^{2} \delta_{1}
\end{aligned}
$$

The right-hand side of (4.13) is positive for $\delta_{1}$ small enough, provided $\delta_{2}=C_{8} \delta_{1}$, with a large enough constant $C_{8}$.

Next we will show that

$$
\mu_{\Delta(B / h),-, T, 0}\left(X_{\Delta(B / h)} \in\left(+m^{*}-\delta_{1},+m^{*}+\delta_{1}\right), \mathscr{F}_{2}\right) \leqq C_{9} \exp \left(-C_{10}(1 / h)^{2-4 b}\right),
$$

where $C_{9}$ and $C_{10}$ depend on $\delta_{1}$ and $\delta_{2}$, but are positive and finite. Because $b<1 / 4$, this estimate is more than enough for our purposes, since in combination with (4.9) it yields

$$
\lim _{h \searrow 0}-h \log \frac{\mu_{\Delta(B / h),-, T, 0}\left(\mathscr{E}_{h, \varepsilon}, X_{\Delta(B / h)} \in\left(m^{*}-\delta_{1}, m^{*}+\delta_{1}\right), \mathscr{F}_{2}\right)}{\mu_{\Delta(B / h),-, T, 0}\left(X_{\Delta(B / h)} \in\left(m^{*}-\delta_{1}, m^{*}+\delta_{1}\right)\right)} e^{|\Delta(B / h)| h \delta_{1}}=+\infty .
$$

We will prove (4.14) by imitating the proof of Lemma 4.1 in [Iof2]. Because our setting is somewhat different from that in that lemma, we will have to reproduce the argument here, rather than being able to just quote the lemma. 
We will denote by $\Omega\left(\Gamma_{1}, \ldots, \Gamma_{k}\right)$ the set of configurations belonging to $\mathscr{F}_{2}$, in which the set of large external contours is $\left\{\Gamma_{1}, \ldots, \Gamma_{k}\right\}$. To prove (4.14), it is enough to show

$$
\mu_{\Delta(B / h),-, T, 0}\left(X_{\Delta(B / h)} \geqq+m^{*}-\delta_{1} \mid \Omega\left(\Gamma_{1}, \ldots, \Gamma_{k}\right)\right) \leqq C_{9} \exp \left(-C_{10}(1 / h)^{2-4 b}\right),
$$

for each $\Omega\left(\Gamma_{1}, \ldots, \Gamma_{k}\right) \in \mathscr{F}_{2}$.

Given $G=\left\{\Gamma_{1}, \ldots, \Gamma_{k}\right\}$, let $\partial_{-} G\left(\right.$ resp. $\left.\partial_{+} G\right)$ be the set of sites where each configuration with the set of external contours equal to $\Gamma_{i}$ is doomed to be-(resp.+). Let $\Lambda_{\text {ext }}$ and $\Lambda_{\text {int }}$ be the components of $\Delta(B / h) \backslash\left(\partial_{-} G \cup \partial_{+} G\right)$ which are, respectively, external and internal to the contours $\Gamma_{i}$. Observe that using (4.11) and the fact that we are on $\mathscr{F}_{2}$ we have

$$
\sum_{i=1}^{n}\left|\gamma_{i}\right| \leqq C_{6} \mathscr{W}(S) \leqq C_{11} / h
$$

From (4.10) it follows now that

$$
\sum_{i=1}^{n}\left|\Gamma_{i}\right| \leqq C_{12}(1 / h)^{2 v} \sum_{i=1}^{n}\left|\gamma_{i}\right| \leqq C_{13}(1 / h)^{1+2 v},
$$

and hence

$$
\left|\partial_{-} G \cup \partial_{+} G\right| \leqq 6 \sum_{l=1}^{n}\left|\Gamma_{i}\right| \leqq C_{14}(1 / h)^{1+2 v}
$$

Because on $\mathscr{F}_{2}$ we have $\hat{V}(S)<\left(1-\left(\delta_{1} / m^{*}\right)\right)(B / h)^{2}$, it follows from (4.12), (4.17) and the fact that $v<1 / 4$ that as $h \searrow 0$,

$$
\left|\Lambda_{\mathrm{nt}}\right| \leqq V(G) \leqq\left(1-\delta_{1} / m^{*}+o(1)\right)(B / h)^{2} .
$$

Because $1+2 v<2$, it follows from (4.18) that for small $h$,

$$
\begin{aligned}
\mu_{\Delta(B / h),-T, 0}\left(X_{\Delta(B / h)}\right. & \left.\geqq m^{*}-\delta_{1} \mid \Omega\left(\Gamma_{1}, \ldots, \Gamma_{k}\right)\right) \\
& \leqq \mu_{\Delta(B / h),-, T, 0}\left(\frac{\left|\Lambda_{\mathrm{int}}\right|}{|\Delta(B / h)|} X_{\Lambda_{\mathrm{ntt}}}+\left(1-\frac{\left|\Lambda_{\mathrm{int}}\right|}{|\Delta(B / h)|}\right) X_{\Lambda_{\mathrm{ext}}}\right. \\
& \left.\geqq m^{*}-\frac{3 \delta_{1}}{2} \mid \Omega\left(\Gamma_{1}, \ldots, \Gamma_{k}\right)\right)
\end{aligned}
$$

Using (4.19) one can check that for the event whose conditional probability is being computed in the right-hand side of (4.20) to happen, one of the following must occur. Either

$$
X_{\Lambda_{\mathrm{ynt}}} \geqq m^{*}+\delta_{1} / 4 \text { and }\left|\Lambda_{\mathrm{int}}\right|>\left(m^{*}-\delta_{1}\right)|\Delta(B / h)| \text {, }
$$

or

$$
X_{\Lambda_{\mathrm{ext}}} \geqq-m^{*}+\delta_{1} / 4
$$


Therefore

$$
\begin{aligned}
& \mu_{\Delta(B / h),-, T, 0}\left(X_{\Delta(B / h)} \geqq m^{*}-\delta_{1} \mid \Omega\left(\Gamma_{1}, \ldots, \Gamma_{k}\right)\right) \\
& \leqq \\
& \quad 1_{\left\{\left|\Lambda_{\mathrm{int}}\right| \geqq\left(m^{*}-\delta_{1}\right)|\Delta(B / h)|\right\}} \mu_{\Delta(B / h),-, T, 0}\left(X_{\Lambda_{\mathrm{int}}} \geqq m^{*}+\delta_{1} / 4 \mid \Omega\left(\Gamma_{1}, \ldots, \Gamma_{k}\right)\right) \\
& \quad+\mu_{\Delta(B / h),-, T, 0}\left(X_{\Lambda_{\mathrm{ext}}} \geqq-m^{*}+\delta_{1} / 4 \mid \Omega\left(\Gamma_{1}, \ldots, \Gamma_{k}\right)\right) .
\end{aligned}
$$

Observe that conditioning on $\Omega\left(\Gamma_{1}, \ldots, \Gamma_{k}\right)$ amounts to conditioning on - spins in $\partial_{-} G$, to + spins in $\partial_{+} G$ and on the absence of large contours in $\Lambda_{\text {ext }}$. Therefore

$$
\begin{aligned}
& \mu_{\Delta(B / h),-, T, 0}\left(X_{\Delta(B / h)} \geqq m^{*}-\delta_{1} \mid \Omega\left(\Gamma_{1}, \ldots, \Gamma_{k}\right)\right) \\
& \leqq \\
& \quad 1_{\left\{\left|\Lambda_{\mathrm{tnt}}\right| \geqq\left(m^{*}-\delta_{1}\right)|\Delta(B / h)|\right\}} \mu_{\Lambda_{\mathrm{lnt}},+, T, 0}\left(X_{\Lambda_{\mathrm{lnt}}} \geqq m^{*}+\delta_{1} / 4\right) \\
& \quad+\mu_{\Lambda_{\mathrm{ext}},-, T, 0}\left(X_{\Lambda_{\mathrm{ext}}} \geqq-m^{*}+\delta_{1} / 4\right),
\end{aligned}
$$

where the superscript $b$ on a Gibbs measure is being used to mean that this Gibbs measure is conditioned to the set of configurations with no large contours.

At this point we can simple use two results in [Iof2]. The first term in the right-hand side of (4.21) is estimated from above, when $\delta_{1}<m^{*}$, by $C_{15} \exp \left(-C_{16}\right.$ $\left.(1 / h)^{2}\right)$, thanks to inequality (4.8) from the proof of Lemma 4.1 in that paper. The second term is estimated from above by $C_{17} \exp \left(-C_{18}(1 / h)^{2-4 b}\right)$, thanks to the fundamental Lemma 3.1 in the same paper (to be able to use this lemma we note the fact that for small $h,\left|\Lambda_{\text {ext }}\right| \geqq\left(\delta_{1} / m^{*}-o(1)\right)(B / h)^{2}$, which follows from (4.18) and (4.19)). (A substantially simplified proof of Lemma 3.1 of [Iof2] is given in the appendix to the present paper.) This completes the proof of (4.14).

Finally we will consider $\mathscr{F}_{3}$ and show that given $\varepsilon>0$, we can choose $\delta_{1}$ and $\delta_{2}$ small enough, so that

$$
\mathscr{E}_{h, \varepsilon} \cap \mathscr{F}_{3}=\emptyset
$$

when $h$ is small. To prove (4.22) we will use the stability theorem for families of curves, with possible self-intersections, proven in Sect. 2.10 of [DKS]. Informally speaking, this theorem assures that if we have a family of curves which surround a total phase volume larger than or equal to 1 , and whose Wulff functionals add up to an amount that is not much larger than the minimum possible, $\beta w$, then there must be a curve in this family which, modulus a translation, is close in Hausdorff distance to a Wulff curve which surrounds a volume 1 . To be able to use this result, we scale down the objects we are studying by a factor $\left(1-\delta_{1} / m^{*}\right)^{1 / 2} B / h$, and use tilde to denote the objects so obtained, i.e., $S=\left\{\gamma_{1}, \ldots, \gamma_{n}\right\}$ is transformed into $\tilde{S}=\left\{\tilde{\gamma}_{1}, \ldots, \tilde{\gamma}_{n}\right\}$. On $\mathscr{F}_{3}$ we have

$$
\hat{V}(\tilde{S}) \geqq 1 \quad \text { and } \quad \mathscr{W}(\tilde{S}) \leqq \frac{1+\delta_{2}}{\left(1-\delta_{1} / m^{*}\right)^{1 / 2}} \beta w .
$$

Theorem 2.10 in $[\mathrm{DKS}]$ tells us then that for some $x \in \mathbb{R}^{2}$ and some $i \in\{1, \ldots, n\}$,

$$
\rho_{H}\left(\tilde{\gamma}_{i}+x, \partial W_{T}\right) \leqq C_{19}\left(\frac{1+\delta_{2}}{\left(1-\delta_{1} / m^{*}\right)^{1 / 2}}-1\right)^{1 / 2}
$$


Blowing the scale up, back again to the original one, we obtain that for some $y \in \mathbb{R}^{2}$,

$$
\begin{aligned}
\rho_{H}\left(\gamma_{i}+y,\left(1-\delta_{1} / m^{*}\right)^{1 / 2}(B / h) \partial W_{T}\right) \\
\quad \leqq C_{19}\left(\frac{1+\delta_{2}}{\left(1-\delta_{1} / m^{*}\right)^{1 / 2}}-1\right)^{1 / 2}\left(1-\delta_{1} / m^{*}\right)^{1 / 2}(B / h) \\
=C_{19}\left(1+\delta_{2}-\left(1-\delta_{1} / m^{*}\right)^{1 / 2}\right)^{1 / 2}(B / h)
\end{aligned}
$$

Using the triangle inequality for $\rho_{H}(\cdot, \cdot)$ and (4.10), it follows that for small $h$,

$$
\begin{aligned}
& \rho_{H}\left(\Gamma_{i}+y, \partial_{\mathrm{ext}} \Delta(B / h)\right) \\
& \quad \leqq\left[C_{19}\left(1+\delta_{2}-\left(1-\delta_{1} / m^{*}\right)^{1 / 2}\right)^{1 / 2}+C_{20}\left(1-\left(1-\delta_{1} / m^{*}\right)^{1 / 2}\right)\right](B / h)+(1 / h)^{v} \\
& \quad \leqq\left[C_{19}\left(1+\delta_{2}-\left(1-\delta_{1} / m^{*}\right)^{1 / 2}\right)^{1 / 2}+C_{21}\left(1-\left(1-\delta_{1} / m^{*}\right)^{1 / 2}\right)\right](B / h) .
\end{aligned}
$$

A little thought convinces one also that before being translated by $y, \Gamma_{i}$ could also not be too far from $\partial_{\mathrm{ext}} \Delta(B / h)$. More precisely, that

$$
\begin{aligned}
& \rho_{H}\left(\Gamma_{i}, \partial_{\mathrm{ext}} \Delta(B / h)\right) \leqq 2 \rho_{H}\left(\Gamma_{i}+y, \partial_{\mathrm{ext}} \Delta(B / h)\right) \\
& \quad \leqq 2\left[C_{19}\left(1+\delta_{2}-\left(1-\delta_{1} / m^{*}\right)^{1 / 2}\right)^{1 / 2}+C_{21}\left(1-\left(1-\delta_{1} / m^{*}\right)^{1 / 2}\right)\right](B / h) .
\end{aligned}
$$

Observe that the quantity which multiplies $1 / h$ in the right-hand side of the last inequality vanishes as $\delta_{1}$ and $\delta_{2}$ both vanish, and that (4.22) must hold in case this quantity is small enough (depending on $\varepsilon$ ), since a chain of-spins cannot cross a contour.

Part (b) of the theorem is obtained by combining (4.8), (4.13), (4.15) and (4.22), and choosing $\delta_{1}$ and $\delta_{2}$ small enough, satisfying the conditions pointed out after (4.13), and in the last paragraph above.

Proof of part (b.3) of Theorem 1. Part (b.3) follows from part (b.2) by a well known argument. First observe that it is enough to prove it for increasing $f$. By the FKG-Holley inequalities, for each $h^{\prime}>0$,

$$
\limsup _{h \searrow 0}\langle f\rangle_{\Delta(B / h),-, T, h} \leqq \limsup _{h \searrow 0}\langle f\rangle_{\Delta(B / h),-, T, h^{\prime}}=\langle f\rangle_{T, h^{\prime}}
$$

Letting now $h^{\prime} \searrow 0$ gives

$$
\underset{h \searrow 0}{\lim \sup }\langle f\rangle_{\Delta(B / h),-, T, h} \leqq\langle f\rangle_{+, T},
$$

since $\mu_{T, h} \rightarrow \mu_{+, T}$ weakly, as $h \searrow 0$. To obtain the complementary bound, observe that for each configuration in $\left(\mathscr{E}_{h, \varepsilon}\right)^{c}$, with $\varepsilon=1 / 2$, say, there is a $\left(^{*}\right)$-circuit of spins + which surrounds $\Delta(B(1-\varepsilon) / h)$, which for small $h$ contains the support of $f$. Partitioning this event according to the outermost such $\left({ }^{*}\right)$-circuit, and using the Markov property and the FKG-Holley inequalities, one obtains the desired inequality from part (b.2).

Proof of Corollary 2. We will first consider the case when $T<T_{c}$ and leave the case $T=T_{c}$ for the end of the proof. 
Let $\mathscr{B}$ be the event that there is a chain of - spins connecting 0 to $\partial_{\text {ext }} \Lambda(l)$. The statement that we want to prove is reduced to an upper estimate on $\mu_{\Lambda(l),-, T, h}(\mathscr{B})$, via a standard argument, which we reproduce next. If $\mathscr{B}$ fails to happen, then there is a $\left(^{*}\right)$-circuit of spins + which surrounds 0 . Partition $\mathscr{B}^{c}$ according to the outermost such circuit and use the notation $\left\{\mathscr{F}_{\alpha}\right\}$ to denote this partition. Using a self-explanatory notation for conditional expectations, from the Markov property and the FKG-Holley inequalities, we obtain for each $\alpha$

Therefore

$$
\left\langle\sigma(0) \mid \mathscr{F}_{\alpha}\right\rangle_{\Lambda(l),-, T, h} \geqq\langle\sigma(0)\rangle_{\Lambda(l),+, T, h} .
$$

$$
\begin{aligned}
& \langle\sigma(0)\rangle_{\Lambda(l),-, T, h} \\
& \quad=\sum_{\alpha}\left\langle\sigma(0) \mid \mathscr{F}_{\alpha}\right\rangle_{\Lambda(l),-, T, h} \mu_{\Lambda(l),-, T, h}\left(\mathscr{F}_{\alpha}\right)+\langle\sigma(0) \mid \mathscr{B}\rangle_{\Lambda(l),-, T, h} \mu_{\Lambda(l),-, T, h}(\mathscr{B}) \\
& \quad \geqq\langle\sigma(0)\rangle_{\Lambda(l),+, T, h} \mu_{\Lambda(l),-, T, h}\left(\mathscr{B}^{c}\right)-\mu_{\Lambda(l),-, T, h}(\mathscr{B}) .
\end{aligned}
$$

Hence

$$
\begin{aligned}
& \langle\sigma(0)\rangle_{\Lambda(l),+, T, h}-\langle\sigma(0)\rangle_{\Lambda(l),-, T, h} \\
& \quad \leqq \mu_{\Lambda(l),-, T, h}(\mathscr{B})+\langle\sigma(0)\rangle_{\Lambda(l),+, T, h} \mu_{\Lambda(l),-, T, h}(\mathscr{B}) \leqq 2 \mu_{\Lambda(l),-, T, h}(\mathscr{B}) .
\end{aligned}
$$

In order to bound $\mu_{\Lambda(l),-T, h}(\mathscr{B})$ from above we will tile $\Lambda(l)$ with square blocks of side $B_{3} / h^{\prime}$, with $B_{3}=B_{3}(T)$ having been defined in Corollary 1 , and $h^{\prime}$ to be chosen later conveniently from the interval $(0, h]$. To be precise (because the lattice is discrete), say that $N_{h^{\prime}}$ is the side of the square $\Lambda\left(B_{3} / h^{\prime}\right)$, and for $i \in \mathbb{Z}^{2}$ use the following notation for the blocks

$$
\Gamma_{i}=\Lambda\left(B_{3} / h^{\prime}\right)+i N_{h^{\prime}} .
$$

Observe that these blocks form a partition of $\mathbb{Z}^{2}$. As usual in blocking arguments, we will think of the blocks as sites of a "renormalized" lattice $\mathbb{Z}^{2}$. In our case we will have to consider percolation on this renormalized lattice, and we will use terminology of the type "a chain of blocks" in a self-explanatory way.

We will use $I(l)$ to denote the set of renormalized sites $i$ for which $\Gamma_{i} \cap \Lambda(l) \neq \emptyset$. Observe that $I(l)$ is a lattice square.

It will be important to consider also larger squares, concentric with the $\Gamma_{i}$. These new squares will be translates of $\Lambda\left(B / h^{\prime}\right)$, where $B$ is a constant larger than the quantity $B_{2}=B_{2}(T)$ defined also in Corollary 1 . We will denote the corresponding square concentric to $\Gamma_{i}$ by $\bar{\Gamma}_{i}$.

We will say that the block $\Gamma_{i}$ is bad in case there is a chain of-spins connecting it to the complement of $\bar{\Gamma}_{i}$; we will use the notation $\mathscr{B}_{i}$ to denote this event. A crucial observation is that if $\mathscr{B}$ happens, then there must exist a chain of bad blocks connecting the origin of the renormalized lattice to the boundary of $I(l)$. To avoid a problem that would affect our estimate if we considered blocks close to the boundary of $I(l)$, we will introduce the notation $\mathscr{B}_{\text {ren }}$ for the event that there exists a chain of bad blocks connecting the origin of the renormalized lattice to some block outside of $I(l / 2)$, and write

$$
\mu_{\Lambda(l),-, T, h}(\mathscr{B}) \leqq \mu_{\Lambda(l),-, T, h}\left(\mathscr{B}_{\text {ren }}\right) .
$$

When $l$ is large, all the squares $\bar{\Gamma}_{l}, i \in I(l / 2)$ are contained in $\Lambda(l)$ (this is the reason for using $I(l / 2)$ rather than $I(l)$ in the definition of $\left.\mathscr{B}_{\text {ren }}\right)$. This allows us 
to use the FKG-Holley inequalities to say the following. Say that the renormalized sites $i$ and $j$ are far from each other if $\bar{\Gamma}_{i} \cap \bar{\Gamma}_{j}=\emptyset$. Given a set $\left\{j_{1}, \ldots, j_{k}\right\} \subset I(l / 2)$ and another renormalized site $i \in I(l)$ such that $i$ is far from $j_{r}$ for all $r \in\{1, \ldots, k\}$,

$$
\mu_{\Lambda(l),-, T, h}\left(\mathscr{B}_{i} \mid \bigcap_{r=1}^{k} \mathscr{B}_{j_{r}}\right) \leqq \mu_{\bar{\Gamma}_{i},-, T, h}\left(\mathscr{B}_{l}\right)=\mu_{\Lambda\left(B / h^{\prime}\right),-, T, h}\left(\mathscr{E}_{h^{\prime}}\right),
$$

where the notation used in the last expression comes from Corollary 1. For arbitrary $h^{\prime} \leqq h$, we can once more use the FKG-Holley inequalities to write the first inequality below; the second inequality is part (b.1) of Corollary 1,

$$
\mu_{\Lambda\left(B / h^{\prime}\right),-, T, h}\left(\mathscr{E}_{h^{\prime}}\right) \leqq \mu_{\Lambda\left(B / h^{\prime}\right),-, T, h}\left(\mathscr{E}_{h^{\prime}}\right) \leqq C e^{-\delta / h^{\prime}}
$$

Inequalities (4.25) and (4.26) allow us to use a type of estimate for finite-rangedependent percolation. First note that each square $\bar{\Gamma}_{i}$ intersects only a fixed number $C_{1}$ of other such squares, where $C_{1}$ may depend on $T$ (through $B$ and $B_{3}$ ), but is independent of $l, h$ and $h^{\prime}$. Given a set of renormalized sites $\left\{i_{1}, \ldots, i_{k}\right\} \subset I(l / 2)$, there is therefore a subset of it with cardinality at least $k /\left(C_{1}+1\right)=k / C_{2}$, so that every one of these sites is far from the others. It follows that

$$
\mu_{\Lambda(l),-, T, h}\left(\bigcap_{r=1}^{k} \mathscr{B}_{i_{r}}\right) \leqq\left(C e^{-\delta / h^{\prime}}\right)^{k / C_{2}} .
$$

There are no more than $4 \cdot 3^{k}$ chains of $k$ renormalized sites having the origin as one of its end-points, and if $\mathscr{B}_{\text {ren }}$ happens, then such a chain must be present, with length at least $k_{0}=(1 / 2)(l / 2) /\left(B_{3} / h^{\prime}\right)$, when $l$ is large. Putting the pieces together we obtain the bound

$$
\mu_{\Lambda(l),-T, h}\left(\mathscr{B}_{\text {ren }}\right) \leqq \sum_{k=k_{0}}^{\infty} 4 \cdot 3^{k}\left(C e^{-\delta / h^{\prime}}\right)^{k / C_{2}}
$$

This is the moment when $h^{\prime}$ has to be chosen. For small enough $h^{\prime}$, this series is summable and yields

$$
\mu_{\Lambda(l),-T, h}\left(\mathscr{B}_{\text {ren }}\right) \leqq C_{3} e^{-\delta k_{0} /\left(C_{2} h^{\prime}\right)}=C_{4} e^{-C_{5} l},
$$

where $C_{5}=\delta /\left(4 B_{3} C_{2}\right)$ does not depend on $h^{\prime}$ and hence does not depend also on $h$. The combination of (4.23), (4.24) and (4.27) completes the proof in case $T<T_{c}$.

Our final task is to address the case $T=T_{c}$. The proof in this case is essentially the same used above, and was left to the end only to simplify the exposition. It should be clear from the proof above that it is enough to verify that given $h>0$ there exists $T<T_{c}$ and $h_{0}^{\prime}>0$ such that for all $0<h^{\prime} \leqq h_{0}^{\prime}$,

$$
\mu_{\Lambda(l),-, T, h^{\prime}} \leqq \mu_{\Lambda(l),-, T_{c}, h} .
$$

That this is indeed the case is a direct consequence of the lemma below.

Lemma 1. For an arbitrary $\Lambda \subset \subset \mathbb{Z}^{2}$ and arbitrary boundary condition $\xi \in \Omega$, if $\left(A+h_{1}\right) / T_{1} \leqq\left(A+h_{2}\right) / T_{2}$ when $A=-4$ and when $A=4$, then

$$
\mu_{\Lambda, \xi, T_{1}, h_{1}} \leqq \mu_{\Lambda, \xi, T_{2}, h_{2}} \text {. }
$$


Proof of Lemma 1. We follow Holley's approach to prove inequalities of this type by running spin-flip time evolutions. The possible time evolutions that we could use are the well known stochastic Ising models (in the terminology of [Lig]), also known as Glauber dynamics. The spin at each site $x \in \Lambda$ flips at time $t$ with a rate which depends on the configuration $\sigma$ where the system is at that time, and is denoted by $c(x, \sigma)$. More precisely, the evolution is a continuous time Markov process with generator $L$ which acts on observables in the following fashion:

$$
(L(f))(\sigma)=\sum_{x \in \Lambda} c(x, \sigma)\left(f\left(\sigma^{x}\right)-f(\sigma)\right),
$$

where $\sigma^{x}$ is the configuration that is identical to $\sigma$ except at the site $x$, where they do differ. The rates $c(x, \sigma)$ are supposed to satisfy certain conditions which assure convergence of the process to the Gibbs measure and moreover allow the comparison that we want to make to be carried on. There are several choices which have these two features, and for no special reason we will take

$$
\begin{aligned}
c(x, \sigma)=c_{T, h}(x, \sigma) & =\exp \left(-\frac{\beta}{2}\left(H_{\Lambda, \xi, h}\left(\sigma^{x}\right)-H_{\Lambda, \xi, h}(\sigma)\right)\right) \\
& =\exp \left(-\frac{\beta}{2} \sigma(x)\left(\sum_{\|y-x\|_{1}=1} \sigma(y)+h\right)\right) .
\end{aligned}
$$

This choice assures reversibility of the Gibbs measure, and by irreducibility the desired convergence to this measure. To obtain the comparison in the lemma, we quote Corollary III.1.5 to Theorem III.1.5 in [Lig]. This corollary states that the desired inequality holds, provided that for all pair of configurations $\eta<\zeta$ and each $x$,

$$
\begin{array}{ll}
c_{T_{1}, h_{1}}(x, \eta) \leqq c_{T_{2}, h_{2}}(x, \eta), & \text { if } \eta(x)=\zeta(x)=-1, \\
c_{T_{1}, h_{1}}(x, \eta) \geqq c_{T_{2}, h_{2}}(x, \eta), & \text { if } \eta(x)=\zeta(x)=+1 .
\end{array}
$$

By translation invariance of the underlying Hamiltonian, there are only finitely many conditions that have to be verified. The inequalities in the last display are actually clearly equivalent to

$$
\frac{A+h_{1}}{T_{1}} \leqq \frac{A+h_{2}}{T_{2}}
$$

for $A=-4,-2,0,2,4$, which are the values that $\sum_{\|y-x\|_{1}=1} \sigma(y)$ can assume. The inequalities with $A=-4$ and $A=4$ imply the other ones by averaging them with appropriate positive coefficients. This completes the proof.

\section{Appendix}

In this appendix we provide a simplified proof of Lemma 3.1 of [Iof2]. This lemma, which is stated below as Lemma A1, refers to Gibbs measures without external field, but conditioned on the absence of large contours. The definition of large contours that we are adopting in this paper is that their volumes are larger than $(1 / h)^{2 b}$, so that $h$ plays a role here. This role is nevertheless just in the form of a scale of length, and we will be using the notation $l=1 / h$ below. We will also use the 
notation $\Omega_{\Lambda,-}^{b}$ to denote the set of configurations in $\Omega_{\Lambda,-}$ in which no large contour is present.

While the proof below seems substantially simpler than that in [Iof2], it was nevertheless inspired by Ioffe's ideas. The main difference is that we do not use the Fortuin-Kastelein random cluster model. The approach in [Sch1] was also an important input for our proof.

Lemma A1. Given $\alpha, \varepsilon>0$, there exist positive and finite constants $C_{1}, C_{2}$ such that if $\Gamma \subset \subset \mathbb{Z}^{2}$ satisfies $|\Gamma| \geqq \alpha l^{2}$, then

$$
\mu_{\Gamma,-, T, 0}^{b}\left(X_{\Gamma} \geqq-m^{*}+\varepsilon\right) \leqq C_{1} \exp \left(-C_{2} l^{2-4 b}\right) \text {. }
$$

Proof. Set $r=\left\lfloor 2 l^{2 b}\right\rfloor$, partition $\mathbb{Z}^{2}$ into the following $r^{2}$ sublattices:

$$
\mathscr{L}_{j}=r \mathbb{Z}^{2}+j, \quad j \in\{0, \ldots, r-1\}^{2}=J,
$$

and partition $\Gamma$ accordingly:

$$
\Gamma_{j}=\Gamma \cap \mathscr{L}_{j}
$$

In case $X_{\Gamma} \geqq-m^{*}+\varepsilon$, there must exist a $j \in J$ such that $\left|\Gamma_{j}\right| \geqq(\varepsilon \alpha / 4)\left(l^{2} / r^{2}\right)$ and $X_{\Gamma_{j}} \geqq-m^{*}+\varepsilon / 2$. To see this, suppose otherwise and note that then

$$
|\Gamma| X_{\Gamma}=\sum_{j \in J}\left|\Gamma_{j}\right| X_{\Gamma_{j}}<\left(-m^{*}+\varepsilon / 2\right) \sum_{j \in J}\left|\Gamma_{j}\right|+\sum_{j \in J}(\varepsilon \alpha / 4)\left(l^{2} / r^{2}\right),
$$

which implies

$$
X_{\Gamma}<\left(-m^{*}+\varepsilon / 2\right)+(\varepsilon \alpha / 4) l^{2} /|\Gamma| \leqq-m^{*}+3 \varepsilon / 4 .
$$

Our estimate will therefore be based on

$$
\mu_{\Gamma,-, T, 0}^{b}\left(X_{\Gamma} \geqq-m^{*}+\varepsilon\right) \leqq \sum_{j \in J} 1_{\left\{\left|\Gamma_{j}\right| \geqq(\varepsilon \alpha / 4)\left(l^{2} / r^{2}\right)\right\}} \mu_{\Gamma,-, T, 0}^{b}\left(X_{\Gamma_{J}} \geqq-m^{*}+\varepsilon / 2\right) .
$$

To estimate each term in the right-hand side of (5.1), we will show that, for each $j \in J$, the probability measure $\mu_{\Gamma,-, T, 0}^{b}$ restricted to $\{-1,+1\}^{\Gamma_{J}}$ is bounded from above, in the sense of (2.2), by a homogeneous product measure $\mathbb{P}$, with marginals that satisfy $\int \sigma(x) d \mathbb{P}=-m^{*}$ for each $x \in \Gamma_{j}$. For this purpose it is well known that it is enough to prove that for all $n$, all distinct $x, y_{1}, \ldots, y_{n} \in \Gamma_{j}$, and every choice of $a_{i} \in\{-1,+1\}$, for $i=1, \ldots, n$,

$$
\left.\mu_{\Gamma,-, T, 0}^{b}\left(\sigma(x)=+1 \mid \sigma\left(y_{i}\right)=a_{i}, i=1, \ldots, n\right) \leqq \mu_{-}(\sigma(0)=+1)\right) .
$$

With $n, x, y_{1}, \ldots, y_{n}$ and $a_{1}, \ldots, a_{n}$ fixed, associate to each configuration $\sigma$ the set $\mathscr{C}(\sigma)$ of sites which can be connected to some site in $\left\{y_{1}, \ldots, y_{n}\right\}$ by a chain of + spins in $\sigma$. Let $\left\{\mathscr{F}_{\alpha}\right\}$ be the partition of $\Omega_{\Gamma,-}^{b}$ according to what $\mathscr{C}$ is, and for each $\alpha$ set

$$
\Gamma(\alpha)=\Gamma \backslash\left(\left\{y_{1}, \ldots, y_{n}\right\} \cup \mathscr{C}(\sigma) \cup \partial_{\mathrm{ext}} \mathscr{C}(\sigma)\right),
$$

where $\sigma$ is any representative of $\mathscr{F}_{\alpha}$. Our choice of $r$ was made having in mind that now we can assure, due also to the definition of large contours, that for each 
$\alpha$ we have $x \in \Gamma(\alpha)$. Moreover there is a sort of "screening phenomenon" taking place; informally speaking the spin at $x$ cannot see any spin +1 which we may possibly have put in some of the sites $y_{i}$, when we chose the $a_{i}$. More precisely, a direct computation based on the definition of the Gibbs measures gives for each $\alpha$,

$$
\mu_{\Gamma,-, T, 0}^{b}\left(\sigma(x)=+1 \mid \sigma\left(y_{l}\right)=a_{i}, i=1, \ldots, n, \mathscr{F}_{\alpha}\right)=\mu_{\Gamma(\alpha),-, T, 0}^{b}(\sigma(x)=+1) .
$$

Given a set $\Lambda \subset \subset \mathbb{Z}^{2}$, to check whether a configuration is in $\Omega_{\Lambda,-}^{b}$ it is, of course, enough to verify that none of the exterior contours is large. Using this remark it is clear that if $\sigma \in \Omega_{\Lambda,-}^{b}$ and $\sigma^{\prime}$ is obtained from $\sigma$ by flipping some spin that was +1 in $\sigma$, then also $\sigma^{\prime} \in \Omega_{\Lambda,-}^{b}$. Therefore we can use the FKG-Holley inequalities to write

$$
\mu_{\Gamma(\alpha),-, T, 0}^{b}(\sigma(x)=+1) \leqq \mu_{\Gamma(\alpha),-, T, 0}(\sigma(x)=+1) .
$$

From (5.3), (5.4) and another application of the FKG-Holley inequalities we obtain now for each $\alpha$,

$$
\mu_{\Gamma,-, T, 0}^{b}\left(\sigma(x)=+1 \mid \sigma\left(y_{i}\right)=a_{i}, i=1, \ldots, n, \mathscr{F}_{\alpha}\right) \leqq \mu_{-, T}(\sigma(0)=+1),
$$

which implies (5.2)

The fact that for each $j \in J$, the probability measure $\mu_{\Gamma,-, T, 0}^{b}$ restricted to $\{-1,+1\}^{\Gamma_{j}}$ is bounded from above, in the sense of $(2.2)$, by a homogeneous product measure $\mathbb{P}$, with marginals that satisfy $\int \sigma(x) d \mathbb{P}=-m^{*}$ for each $x \in \Gamma_{j}$, leads to the following standard large deviation estimate

$$
\begin{aligned}
1_{\left\{\left|\Gamma_{j}\right| \geqq(\varepsilon \alpha / 4)\left(l^{2} / r^{2}\right)\right\}} & \mu_{\Gamma,-, T, 0}^{b}\left(X_{\Gamma_{j}} \geqq-m^{*}+\varepsilon / 2\right) \\
& \leqq C_{3} \exp \left(-C_{4} l^{2} / r^{2}\right)=C_{3} \exp \left(-C_{5} l^{2-4 b}\right) .
\end{aligned}
$$

Combining (5.1) and (5.5) we finish the argument, since the cardinality of the set $J$ grows only as a power of $l$.

Acknowledgements. We thank D. Ioffe for sending us copies of [Iof2] prior to publication. The idea of using a type of FKG-Holley comparison involving different temperatures, as in Lemma 1, to extend Corollary 2 to the case $T=T_{c}$, was inspired by recollections of conversations that R.H.S. had with F. Martinelli at the time that [MOS] was being written.

\section{References}

[AR] Abraham, D.B., Reed, P.: Diagonal interface in the two-dimensional Ising ferromagnet. J. Phys. A: Math. Gen. 10, L121-L123 (1977)

[CCS] Chayes, J., Chayes, L., Schonmann, R.H.: Exponential decay of connectivities in the twodimensional Ising model. J. Stat. Phys. 49, 433-445 (1987)

[DKS] Dobrushin, R.L., Kotecký, R., Shlosman, S.: Wulff construction: A global shape from local interaction. AMS translation series, 1992

[DS1] Dobrushin, R.L., Shlosman, S.: Constructive criterion for the uniqueness of Gibbs fields. In: Statistical Physics and Dynamical Systems, Fritz, J., Jaffe, A., Szász, D., ed., BaselBoston: Birkhauser, pp. 347-370

[DS2] Dobrushin, R.L., Shlosman, S.: Completely analytical Gibbs fields. In: Statistical Physics and Dynamical Systems, Fritz, J., Jaffe, A., Szász, D., ed., Basel, Boston: Birkhauser, pp. $371-403$

[DS3] Dobrushin, R.L., Shlosman, S.: Completely analytical interactions. Constructive description. J. Stat. Phys. 46, 983-1014 (1987) 
[FO] Föllmer, H., Orey, S.: Large deviations for the empirical field of a Gibbs measure. Ann. Prob. 16, 961-977 (1988)

[Hi] Higuchi, Y.: Coexistence of infinite $\left({ }^{*}\right)$-clusters II:- Ising percolation in two dimensions. Prob. Theory and Related Fields 97, 1-34 (1993)

[lof1] Ioffe, D.: Large deviations for the 2D Ising model: A lower bound without cluster expansions. J. Stat. Phys. 74, 411-432 (1994)

[lof2] Ioffe, D.: Exact large deviation bounds up to $T_{c}$ for the Ising model in two dimensions. Preprint

[Lan] Lanford, O.E.: Entropy and equilibrium states in classical statistical mechanics. Lecture Notes in Physics, vol. 20, Berlin-Heidelberg-New York: Springer, 1973, pp. 1-113

[Lig] Liggett, T.: Interacting Particle Systems, Berlin, Heidelberg. New York: Springer

[LY] Lu, S.L., Yau, H.T.: Spectral gap and logarithmic Sobolev inequality for Kawasaki and Glauber dynamics. Commun. Math. Phys. 156, 399-433 (1993)

[Mar] Martirosyan, D.G.: Theorems on strips in the classical Ising ferromagnetic model. Sov. J. Contemp. Math. Anal. 22, 59-83 (1987)

[MO1] Martinelli, F., Olivieri, E.: Approach to equilibrium of Glauber dynamics in the one phase region I. The attractive case. Commun. Math. Phys. 161, 447-486 (1994)

[MO2] Martinelli, F., Olivieri, E.: Approach to equilibrium of Glauber dynamics in the one phase region II. The general case. Commun. Math. Phys. 161, 487-514 (1994)

[MOS] Martinelli, F., Olivieri, E., Schonmann, R.H.: For 2-D lattice spin systems weak mixing implies strong mixing. Commun. Math. Phys., 165, 33-47 (1994)

[Oll] Olla, S.: Large deviations for Gibbs random fields, Prob. Th. Rel. Fields 77, 343-357 (1988)

[Pfi] Pfister, C.E.: Large deviations and phase separation in the two-dimensional Ising model. Helv. Phys. Acta 64, 953-1054 (1991)

[Sch1] Schonmann, R.H.: Exponential convergence under mixing. Prob. Th. Rel. Fields 81, 235-238 (1989)

[Sch2] Schonmann, R.H.: Slow droplet-driven relaxation of stochastic Ising models in the vicinity of the phase coexistence region. Commun. Math. Phys. 161, 1-49 (1994)

[SS] Schonmann, R.H., Shlosman, S.B.: Constrained variational problem with applications to the Ising model. In preparation

[Sh1] Shlosman, S.B.: Uniqueness and half-space nonuniqueness of Gibbs states in Czech model. Theor. Math. Phys. 66, 284-293 (1986)

[SZ] Stroock, D.W., Zegarlinski, B.: The logarithmic Sobolev inequality for Discrete spin systems on a lattice. Commun. Math. Phys. 149, 175-194 (1992) 